\title{
Optimal probes and error-correction schemes in multi-parameter quantum metrology
}

\author{
Wojciech Górecki*1 ${ }^{2}$ Sisi Zhou*2,3,4, Liang Jiang ${ }^{2,3,4}$, and Rafał Demkowicz-Dobrzański ${ }^{1}$ \\ ${ }^{1}$ Faculty of Physics, University of Warsaw, Pasteura 5, 02-093 Warsaw, Poland \\ ${ }^{2}$ Departments of Applied Physics and Physics, Yale University, New Haven, Connecticut 06511, USA \\ ${ }^{3}$ Yale Quantum Institute, Yale University, New Haven, Connecticut 06511, USA \\ ${ }^{4}$ Pritzker School of Molecular Engineering, University of Chicago, Chicago, IL 60637, USA
}

We derive a necessary and sufficient condition for the possibility of achieving the Heisenberg scaling in general adaptive multi-parameter estimation schemes in presence of Markovian noise. In situations where the Heisenberg scaling is achievable, we provide a semidefinite program to identify the optimal quantum error correcting (QEC) protocol that yields the best estimation precision. We overcome the technical challenges associated with potential incompatibility of the measurement optimally extracting information on different parameters by utilizing the Holevo Cramér-Rao (HCR) bound for pure states. We provide examples of significant advantages offered by our joint-QEC protocols, that sense all the parameters utilizing a single error-corrected subspace, over separate-QEC protocols where each parameter is effectively sensed in a separate subspace.

\section{Introduction}

Quantum metrology aims at exploiting all possible features of quantum systems, such as coherence or entanglement, in order to boost the precision of measurements beyond that achievable by metrological schemes that operate within classical or semi-classical paradigms [1-9]. The most persuasive promise of quantum metrology is the possibility of obtaining the so-called Heisenberg scaling (HS), which manifests itself in the quadratically improved scaling of precision as a function of number of elementary probe systems involved in the experiment [10-19] or the total interrogation time of a probe system [20]. In either of these cases, the presence of decoherence typically restricts the quadratic improvement to a small particle number or a short-time regime, whereas in the asymptotic regime the quantum-enhancement amounts to constant factor improvements [21-26] even under the most general adaptive schemes [27]. Still, there are specific models where even in the presence of decoherence the asymptotic HS is achievable via application of appropriate quantum error correction (QEC) protocols [28-42].

Recently, a general theory providing a necessary and sufficient condition, the HNLS condition (an acronym for "Hamiltonian-Not-in-Lindblad-Span"), for achieving the HS in a finite-dimensional system in the most general adaptive quantum metrological protocols under Markovian noise, has been developed $[35,36]$. The theory allows for a quick identification of the most promising quantum metrological models and provides a clear recipe for designing the optimal adaptive schemes based on appropriately tailored QEC protocols. However, HNLS is restricted to the single-parameter estimation case, while a lot of relevant metrological problems, like vector field sensing (e.g. magnetic field) [43], imaging [44], multiple-arm interferometry [45, 46] or waveform estimation [47, 48] are inherently multi-parameter estimation problems. Multi-parameter estimation problems drew a lot of attention in recent years [49-56], yet the fundamental questions regarding the achievability of the HS as well as the form of the optimal metrological protocols in multiple-parameter estimation in presence of noise have not been answered so far. The aim of this paper is to fill this gap.

*These two authors provided key and equal contributions to the project. 


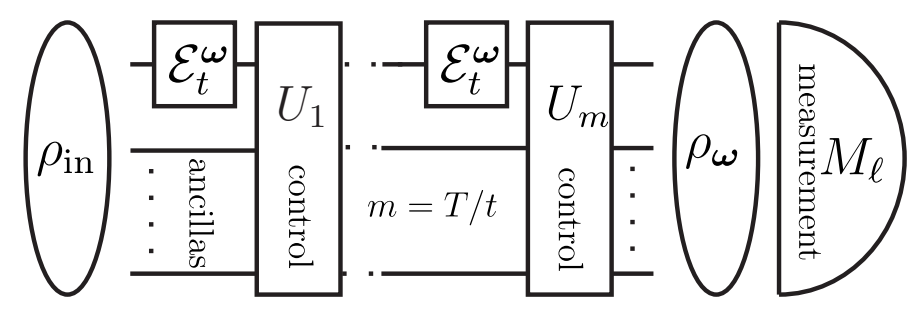

Figure 1: General adaptive mutli-parameter quantum metrological scheme, where $P$ parameters $\boldsymbol{\omega}=\left[\omega_{i}\right]_{i=1}^{P}$ are to be estimated. Total probe system evolution time $T$ is divided into a number $m$ of $t$-long steps of probe evolution $\mathcal{E}_{t}^{\omega}$ interleaved with general unitary controls $U_{i}$. In the end a general POVM $\left\{M_{\ell}\right\}$ is performed yielding estimated value of all parameters $\tilde{\boldsymbol{\omega}}(\ell)$ with probability $p(\ell)=\operatorname{Tr}\left(\rho_{\boldsymbol{\omega}} M_{\ell}\right)$.

The methods developed for the single parameter estimation case, in particular the semidefinite program (SDP) that allows to identify the optimal QEC protocol [36], are not applicable in the multi-parameter regime. The reasons are threefold.

First, the widely used quantum Cramér-Rao (CR) bound for multiple parameters is not in general saturable, due to the incompatibility of the optimal measurements for different parameters $[49,51,52]$. Therefore, unlike in the single-parameter case, the quantum Fisher information (QFI) does not provide the full insight into the problem [51, 57-60]. On the other hand, stronger bounds, such as the HCR bound [59-64], have no closed-form expressions (except for specific cases [65]). Moreover, the HCR bound, although solvable via an SDP [66], does not shed light on the corresponding optimal measurements saturating it. In general, the HCR bound is only saturable when collective measurements on all copies of quantum states are allowed, which is a demanding condition in practice $[63,64]$. As a result, the optimal measurements on the output quantum states are hard to identify.

Second, there is no general recipe to find the optimal input states in multi-parameter estimation even in the noiseless case [52, 53], unlike in the single-parameter estimation case where the optimal input state is simply the equally weighted superposition between the eigenstates corresponding to the maximum and minimum eigenvalues of the Hamiltonian.

Finally, in the single-parameter case [36], all valid two-dimensional QEC codes were mapped into a traceless Hermitian matrix representing the difference between logical zero and one codes. In the multi-parameter case, however, it is not clear whether valid QEC codes could be mapped into a convex set when the code dimension is large, which is inevitable in multi-parameter estimation.

In this paper, we generalize the HNLS condition to multi-parameter scenarios, and provide an SDP to find the best possible QEC protocol in the situations when the HNLS condition is satisfied (including all noiseless cases). The solution yields an explicit form of the optimal input state, QEC codes and measurements. No collective measurements are required on the output states. Our protocol goes beyond the typically used QFI-based formalism and overcomes all the challenges related with the multi-parameter aspect of the problem mentioned above. Our work reveals the advantage of QEC protocols in multi-parameter estimation and we expect that the SDP formulation of our problem will also be an inspiration for other research areas in quantum error correction and quantum metrology.

\section{Formulation of the model}

We assume the dynamics of a $d$-dimensional probe system $\mathcal{H}_{S}$ is given by a general quantum master equation [67-69]:

$$
\frac{d \rho}{d t}=-i[H, \rho]+\sum_{k=1}^{r}\left(L_{k} \rho L_{k}^{\dagger}-\frac{1}{2}\left\{L_{k}^{\dagger} L_{k}, \rho\right\}\right)
$$

where the parameters to be estimated $\boldsymbol{\omega}=\left[\omega_{1}, \ldots, \omega_{P}\right]$ enter linearly into the Hamiltonian of the evolution via Hermitian generators $\boldsymbol{G}=\left[G_{1}, \ldots, G_{P}\right]^{T}$ (where ${ }^{T}$ denotes transpose) so that $H=\boldsymbol{\omega} \cdot \boldsymbol{G} \equiv \sum_{k=1}^{P} \omega_{k} G_{k}$, and $L_{k}$ are operators representing a general Markovian noise. Similar to the previous investigations [35, 36] we consider the most general adaptive scheme (see Fig. 1) [27] 
with an unlimited number of ancillae (denoted jointly as $\mathcal{H}_{A}$ ), instantaneous perfect intermediate unitary operations $U_{i}$ and a general POVM on the final state $\rho_{\boldsymbol{\omega}} \cdot \mathcal{E}_{t}^{\boldsymbol{\omega}}$ represents the probe system dynamics integrated over time $t$, whereas the total probe interrogation time is $T$. Such schemes are the most general schemes of probing quantum dynamics, assuming the total interrogation time is $T$, and encompass in particular all QEC procedures.

In single-parameter estimation the optimal protocol is the one that yields the minimum estimation variance. In multi-parameter case the estimator covariance matrix is the key object capturing estimation precision, defined as [58, 59]:

$$
\Sigma_{i j}=\sum_{\ell} \operatorname{Tr}\left(\rho_{\boldsymbol{\omega}} M_{\ell}\right)\left(\tilde{\omega}_{i}(\ell)-\omega_{i}\right)\left(\tilde{\omega}_{j}(\ell)-\omega_{j}\right)
$$

for $i, j=1, \ldots, P$, where $M_{\ell} \geq 0, \sum_{\ell} M_{\ell}=\mathbb{1}$, are measurement operators (" $\geq 0$ " for matrices means positive semidefinite) and $\tilde{\boldsymbol{\omega}}(\ell)$ is an estimator function mapping a measurement result $\ell$ to the parameter space.

Diagonal entries of $\Sigma$ represent variances of estimators of respective parameters while offdiagonal terms represent covariance between different parameters. As a figure of merit one may simply choose $\operatorname{Tr}(\Sigma)$ which will be the sum of all individual parameter variance, or more generally $\operatorname{Tr}(W \Sigma)$, where $W$ is a real positive cost matrix that determines the weight we associate with each parameter in the effective scalar cost function

$$
\Delta_{W}^{2} \tilde{\boldsymbol{\omega}} \equiv \operatorname{Tr}(W \Sigma) .
$$

Note that we require strict positivity of $W$ which is equivalent to saying that it is an estimation problem of all $P$ parameters, and not a problem where effectively only a smaller number of parameters are relevant. We assume the measurement-estimation strategy to be locally unbiased at some fixed parameter point $\boldsymbol{\omega}$, i.e.

$$
\sum_{\ell} \tilde{\omega}_{j}(\ell) \operatorname{Tr}\left(\rho_{\boldsymbol{\omega}} M_{\ell}\right)=\omega_{j}, \quad \sum_{\ell} \tilde{\omega}_{j}(\ell) \operatorname{Tr}\left(\partial_{i} \rho_{\boldsymbol{\omega}} M_{\ell}\right)=\delta_{i j}
$$

where $\partial_{i} \rho_{\boldsymbol{\omega}}=\frac{\partial \rho_{\omega}}{\partial \omega_{i}}$, which is a standard assumption necessary to obtain meaningful precision bounds within the frequentist estimation framework [70, 71].

\section{The necessary and sufficient condition for the HS}

We say that the HS in a multi-parameter estimation problem is achieved when there exists an adaptive protocol such that for every $W>0, \Delta_{W}^{2} \tilde{\boldsymbol{\omega}} \propto 1 / T^{2}$ in the limit $T \rightarrow \infty$. This is equivalent to a requirement that all parameters (and any combination of parameters) are estimated with precision that scales like the HS. The following theorem generalize the HNLS condition $[35,36]$ to multi-parameter scenarios.

Theorem 1 (Multi-parameter HNLS). The HS can be achieved in a multi-parameter estimation problem if and only if $\left\{\left(G_{i}\right)_{\perp}, i=1, \ldots, P\right\}$ are linearly independent operators. Here $\left(G_{i}\right)_{\perp}$ are orthogonal projections of $G_{i}$ onto space $\mathcal{S}^{\perp}$ which is the orthogonal complement of the Lindblad span

$$
\mathcal{S}=\operatorname{span}_{\mathbb{R}}\left\{\mathbb{1}, L_{k}^{\mathrm{H}}, i L_{k}^{\mathrm{AH}},\left(L_{k}^{\dagger} L_{j}\right)^{\mathrm{H}}, i\left(L_{k}^{\dagger} L_{j}\right)^{\mathrm{AH}}, \forall j, k\right\},
$$

in the Hilbert space of Hermitian matrices under the standard Hilbert-Schmidt scalar product, whereas the superscripts ${ }^{\mathrm{H}},{ }^{\mathrm{AH}}$ denote the Hermitian and anti-Hermitian part of an operator respectively.

Proof. Let us start with a brief review of the HNLS condition in the single-parameter case, where $H=\omega G$ involves only a single generator $G$. As shown in [35, 36], the necessary and sufficient condition to achieve the HS is that $G \notin \mathcal{S}$, or in other words that $G_{\perp} \neq 0$. In particular, following [36] (see the section named "QEC code for HL scaling when HNLS holds"), an explicit construction of the optimal QEC code was provided, where the code space $\mathcal{H}_{\mathcal{C}} \subseteq \mathcal{H}_{S} \otimes \mathcal{H}_{A}$ is defined on the Hilbert space of the probe system $\mathcal{H}_{S}$ extended by an ancillary space $\mathcal{H}_{A} \cong \mathcal{H}_{S}$. The code space satisfies the QEC condition [36, 72]:

$$
\Pi_{\mathcal{H}_{\mathcal{C}}}(S \otimes \mathbb{1}) \Pi_{\mathcal{H}_{\mathcal{C}}} \propto \Pi_{\mathcal{H}_{\mathcal{C}}}, \forall S \in \mathcal{S},
$$


where the operator $S$ acting on $\mathcal{H}_{S}$ was tensored with identity on $\mathcal{H}_{A}$ and $\Pi_{\mathcal{H}_{\mathcal{C}}}$ denotes the projection onto $\mathcal{H}_{\mathcal{C}}$. Metrological sensitivity is guaranteed by the fact that $G$ acts non-trivially on $\mathcal{H}_{\mathcal{C}}:$

$$
G^{\mathcal{C}}=\Pi_{\mathcal{H}_{\mathcal{C}}}(G \otimes \mathbb{1}) \Pi_{\mathcal{H}_{\mathcal{C}}} \not \subset \Pi_{\mathcal{H}_{\mathcal{C}}} .
$$

As a result we obtain a noiseless unitary evolution generated by $G^{\mathcal{C}}$ leading to the HS in the estimation precision of $\omega$.

(Necessity) Suppose $\left(G_{i}\right)_{\perp}$ 's are linearly dependent. Then there exists a linear (invertible) transformation on the parameter space $A \in \mathbb{R}^{P \times P}: \boldsymbol{\omega}^{\prime}=\boldsymbol{\omega} A^{-1}$, (where we also modify accordingly the generators $\boldsymbol{G}^{\prime}=A \boldsymbol{G}$ and the cost matrix $W^{\prime}=A W A^{T}$, so that $H$ and $\Delta_{W}^{2} \tilde{\boldsymbol{\omega}}$ remain unchanged), such that $\left(G_{i}^{\prime}\right)_{\perp}=0$ for some $i$. Then, from the single-parameter theorem, $\omega_{i}^{\prime}$ cannot be estimated with precision better than $\Delta^{2} \tilde{\omega}_{i}^{\prime} \sim 1 / T$ which contradicts the HS requirements.

(Sufficiency) Suppose $\left(G_{i}\right)_{\perp}$ 's are linearly independent. We assume the ancillary space to be a direct sum of $P$ subspaces $\mathcal{H}_{A_{i}}$ so that the whole Hilbert space is $\mathcal{H}_{S} \otimes\left(\mathcal{H}_{A_{1}} \oplus \cdots \oplus \mathcal{H}_{A_{P}}\right)$ (see Fig. 2a). We may construct separate code spaces for each parameter using orthogonal ancillary subspace $\mathcal{H}_{\mathcal{C}_{i}} \subseteq \mathcal{H}_{S} \otimes \mathcal{H}_{A_{i}}$ so that the QEC conditions Eq. (6) are satisfied within each code space $\mathcal{H}_{\mathcal{C}_{i}}$ separately. While constructing the code space for the $i$-th parameter, we include all the remaining generators $G_{j}(j \neq i)$ in the Lindblad span, so effectively treating them as noise i.e. $\mathcal{S}_{i}=\operatorname{span}_{\mathbb{R}}\left\{\left\{\mathbb{1}, L_{k}^{\mathrm{H}}, i L_{k}^{\mathrm{AH}},\left(L_{k}^{\dagger} L_{j}\right)^{\mathrm{H}}, i\left(L_{k}^{\dagger} L_{j}\right)^{\mathrm{AH}}\right\}_{j, k} \cup\left\{G_{j}\right\}_{j \neq i}\right\}$. As a result thanks to the QEC condition it follows that $\forall_{i \neq j} \Pi_{\mathcal{H}_{\mathcal{C}_{i}}}\left(G_{j} \otimes \mathbb{1}\right) \Pi_{\mathcal{H}_{\mathcal{c}_{i}}} \propto \Pi_{\mathcal{H}_{\mathcal{C}_{i}}}$ and hence within a given subspace only one parameter is being sensed via the effective generator $G_{i}^{\mathcal{C}_{i}}=\Pi_{\mathcal{H}_{\mathcal{C}_{i}}}\left(G_{i} \otimes \mathbb{1}\right) \Pi_{\mathcal{H}_{\mathcal{C}_{i}}}$, while all other generators act trivially. If $\left|\psi_{i}\right\rangle \in \mathcal{H}_{\mathcal{C}_{i}}$ is the optimal state for measuring $\omega_{i}$, the state to be used in order to obtain HS for all parameters which is not affected by noise reads $\rho_{\text {in }}=\frac{1}{P} \sum_{i=1}^{P}\left|\psi_{i}\right\rangle\left\langle\psi_{i}\right| \in$

$\mathcal{H}_{S} \otimes\left(\bigoplus_{i=1}^{P} \mathcal{H}_{A_{i}}\right)$ - there is no measurement incompatibility issue because different parameters are encoded on orthogonal subspaces.

Similar to the single-parameter case, it must be admitted that in realistic situations with generic noise, HNLS is often violated [24, 35]. Therefore, a more pragmatic approach is required taking into account the fact that in a real experiment the total time of evolution $T$ is always finite. Let us consider a situation where $H \in \mathcal{S}$, but where some noise components are weak [36]. Specifically, we divide Lindblad operators in Eq. (1) into two sets - strong noise generators $\left\{L_{k}\right\}$ and weak noise generators $\left\{J_{m}\right\}$ satisfying $\epsilon:=\left\|\sum_{m} J_{m}^{\dagger} J_{m}\right\|$ where $\|\cdot\|$ denotes operator norm. If the HNLS condition is satisfied for the strong noise part, we could choose the code space $\mathcal{H}_{\mathcal{C}}$ which allows to completely erase the strong noise $\left\{L_{k}\right\}$ and the resulting effective noise rate would be upper bounded by $\epsilon$ [36]. This means that the distance between state of the error-corrected probe and the state evolving under ideal noiseless evolution will be of the order $\Theta(\epsilon T)$. Therefore, for sufficiently short evolution, $T=o(1 / \epsilon)$, the precision of estimation will still scale quadratically with the total time $\Delta_{W}^{2} \tilde{\boldsymbol{\omega}} \propto \frac{1}{T^{2}}$ whereas for larger $T$, it will gradually approach the standard $1 / T$ scaling.

\section{Optimal probes, error correction schemes and measurements}

In Sec. 3, we provided a QEC code where each parameter is sensed separately in different errorcorrected subspaces (see Fig. 2a). Such protocols will be referred as separate-QEC protocols (SEP-QEC). In contrast to this construction, we will now consider QEC strategies which allow for simultaneous estimation of all the parameters in a single coherent protocol by utilizing states within a single protected code space, which we will call the joint-parameter QEC scheme (JNTQEC). In this section we provide a general method to identify the optimal JNT-QEC, while its potential advantages over the optimal SEP-QEC will be discussed in Sec. 5, as well as in Sec. 6 when studying concrete estimation models.

From now on, we assume the multi-parameter HNLS condition is satisfied. Without loss of generality, we assume the generators $\left\{G_{i}\right\}_{i=1}^{P} \subset \mathcal{S}^{\perp}$ are orthonormal, since the components in $\mathcal{S}$ do not contribute and there is always a linear transformation $A$ on parameters leading to orthonormality. The following theorem provides a recipe to find the optimal JNT-QEC.

Theorem 2 (Optimal JNT-QEC). Given a cost matrix $W$. If the multi-parameter HNLS condition is satisfied with generators $\left\{G_{i}\right\}_{i=1}^{P} \subset S^{\perp}$, the minimum cost $\Delta_{W}^{2} \tilde{\boldsymbol{\omega}}$ that can be achieved in a JNT- 
(a)

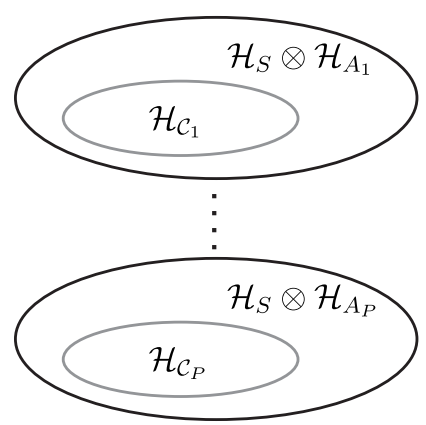

(b)

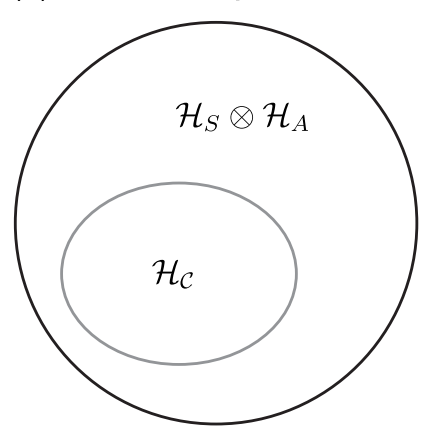

(c)

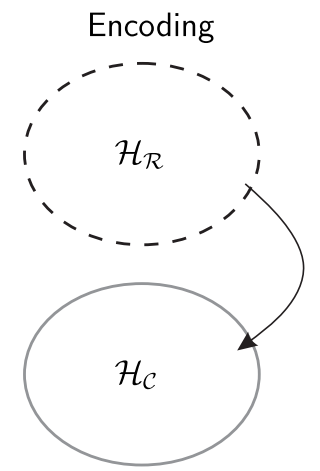

Figure 2: Schematic diagrams of relations between Hilbert spaces $\mathcal{H}_{S}, \mathcal{H}_{A}, \mathcal{H}_{\mathcal{C}}, \mathcal{H}_{A_{i}}, \mathcal{H}_{\mathcal{C}_{i}}, \mathcal{H}_{\mathcal{R}}$. (a) In SEPQEC, we use $P$ mutually orthogonal ancillary subspaces $\mathcal{H}_{A_{i}}$ to sense each parameter $\omega_{i}$. $\mathcal{H}_{A}=\bigoplus_{i=1}^{P} \mathcal{H}_{A_{i}}$ and $\mathcal{H}_{\mathcal{C}}=\bigoplus_{i=1}^{P} \mathcal{H}_{\mathcal{C}_{i}}$. $\operatorname{dim}\left(\mathcal{H}_{A_{i}}\right)=\operatorname{dim}\left(\mathcal{H}_{S}\right)=d$ and $\operatorname{dim}\left(\mathcal{H}_{\mathcal{C}_{i}}\right)=2$. (b) In JNT-QEC, we use a single code space $\mathcal{H}_{\mathcal{C}} \subseteq \mathcal{H}_{S} \otimes \mathcal{H}_{A}$ to estimate all parameters jointly. $\operatorname{dim}\left(\mathcal{H}_{A}\right)=(P+1) d$ and $\operatorname{dim}\left(\mathcal{H}_{\mathcal{C}}\right)=P+1$. (c) We use $\mathcal{H}_{\mathcal{R}}$ to represent the logical space $\operatorname{span}\{|0\rangle,|1\rangle, \ldots,|P\rangle\}$ which is encoded into the physical space $\mathcal{H}_{\mathcal{C}}=\operatorname{span}\left\{\left|c_{0}\right\rangle,\left|c_{1}\right\rangle, \ldots,\left|c_{P}\right\rangle\right\}$.

QEC reads

$$
\begin{aligned}
& \Delta_{W}^{2} \tilde{\boldsymbol{\omega}}=\frac{P}{4 T^{2}} \min _{G_{i}^{\mathcal{R}}, B_{i}, \nu_{i}, K, w} w, \\
& \text { subject to } \quad \mathbb{1}_{P+1} \otimes \frac{\mathbb{1}_{d}}{d}+\sum_{i=1}^{P}\left(G_{i}^{\mathcal{R}}\right)^{T} \otimes G_{i}+\sum_{i=P+1}^{P^{\prime}} \nu_{i} \mathbb{1}_{P+1} \otimes S_{i}+\sum_{i=P^{\prime}+1}^{d^{2}-1} B_{i} \otimes R_{i} \geq 0 \text {, } \\
& \Gamma_{i j}=\operatorname{Im}\left[G_{j}^{\mathcal{R}}\right]_{i 0}, \quad\left(\begin{array}{cc}
w \mathbb{1}_{P} & K \\
K & \mathbb{1}_{P}
\end{array}\right) \geq 0, \quad\left(\begin{array}{cc}
K & \mathbb{1}_{P} \\
\mathbb{1}_{P} & \Gamma \sqrt{W^{-1}}
\end{array}\right) \geq 0,
\end{aligned}
$$

where $\mathbb{1}_{d} / \sqrt{d},\left\{G_{i}\right\}_{i=1}^{P},\left\{S_{i}\right\}_{i=P+1}^{P^{\prime}},\left\{R_{i}\right\}_{i=P^{\prime}+1}^{d^{2}-1}$ form an orthonormal basis of Hermitian operators acting on $\mathcal{H}_{S}$ such that $\mathcal{S}=\operatorname{span}_{\mathbb{R}}\left\{\mathbb{1}_{d},\left(S_{i}\right)_{i=P+1}^{P^{\prime}}\right\}$. Moreover, $G_{i}^{\mathcal{R}}, B_{i}$ are Hermitian $(P+1) \times$ $(P+1)$ matrices (with matrix indices taking values from 0 to $P$ ), and $\nu_{i} \in \mathbb{R} . \Gamma$ and $K$ are real $P \times P$ matrices (with matrix indices from 1 to $P$ ).

As a semidefinite program (SDP) it could be easily solved numerically, for example, using the Matlab-based package CVX [73]. Before giving a formal proof, let us briefly review some existing bounds in multi-parameter metrology and discuss their saturability.

\subsection{General bounds in multi-parameter metrology}

In this section, we discuss bounds for general parameter estimation problems on fixed quantum states. We use $\mathcal{H}$ to denote a general finite-dimensional Hilbert space.

Most commonly, quantum multi-parameter estimation problems are analyzed utilizing the standard quantum CR bound [57-59]:

$$
\Delta_{W}^{2} \tilde{\boldsymbol{\omega}} \geq \operatorname{Tr}\left(W F^{-1}\right), \quad F_{i j}=\operatorname{Re}\left(\operatorname{Tr}\left(\rho_{\omega} \Lambda_{i} \Lambda_{j}\right)\right),
$$

where $F$ is a $P \times P$ QFI matrix and $\Lambda_{i}$ (symmetric logarithmic derivatives) satisfy $\partial_{i} \rho_{\boldsymbol{\omega}}=\frac{1}{2}\left(\Lambda_{i} \rho_{\boldsymbol{\omega}}+\right.$ $\left.\rho_{\omega} \Lambda_{i}\right)$. This bound is not saturable in general, due to potential non-compatibility of the optimal measurements, unless $\operatorname{Im}\left[\operatorname{Tr}\left(\rho_{\boldsymbol{\omega}} \Lambda_{i} \Lambda_{j}\right)\right]=0$ [51]. Moreover, a direct minimization of the CR bound over all JNT-QEC, with the saturability constraint imposed, does not necessarily guarantee the identification of the optimal protocol - there is a possibility that the optimal protocol does not meet the saturability constraint for the CR bound.

Therefore, in order to identify truly optimal protocols we need to resort to a stronger HCR 
bound $[59,61,62]$ :

$$
\begin{aligned}
& \Delta_{W}^{2} \tilde{\boldsymbol{\omega}} \geq \min _{\left\{X_{i}\right\}}(\operatorname{Tr}(W \cdot \operatorname{Re} V)+\operatorname{Tr}[\operatorname{abs}(W \cdot \operatorname{Im} V)]), \quad \text { where } V_{i j}=\operatorname{Tr}\left(X_{i} X_{j} \rho_{\boldsymbol{\omega}}\right), \\
& \text { for Hermitian } X_{i} \in \mathcal{L}(\mathcal{H}), \quad \text { subject to } \operatorname{Tr}\left(X_{i} \partial_{j} \rho_{\boldsymbol{\omega}}\right)=\delta_{i j},
\end{aligned}
$$

where $\mathcal{L}(\circ)$ denotes the set of all linear operators acting on $\circ$, Re and Im denote the real and imaginary part of a matrix (not to be confused with the Hermitian and anti-Hermitian part of a matrix), and $\operatorname{Tr}[\operatorname{abs}(\cdot)]$ is the sum of absolute values of the eigenvalues of a matrix. When the second term is dropped the HCR bound reduces to the standard CR bound [51, 59]. Unlike the CR bound this bound is saturable in general using collective measurements on many copies [63, 64]. On the other hand, the HCR is defined via an optimization problem, making it usually more difficult to deal with than the standard quantum CR bound with a closed-form expression.

In the case of pure states $\rho_{\boldsymbol{\omega}}=\left|\psi_{\boldsymbol{\omega}}\right\rangle\left\langle\psi_{\boldsymbol{\omega}}\right|$, however, we note that the HCR is exactly equivalent to [49]:

$$
\begin{aligned}
& \Delta_{W}^{2} \tilde{\boldsymbol{\omega}} \geq \min _{\left\{\left|x_{i}\right\rangle\right\}} \operatorname{Tr}(W V), \text { where } V_{i j}=\left\langle x_{i} \mid x_{j}\right\rangle, \\
& \text { for }\left|x_{i}\right\rangle \in \operatorname{span}\left\{\left|\psi_{\boldsymbol{\omega}}\right\rangle, \partial_{1}\left|\psi_{\boldsymbol{\omega}}\right\rangle, \ldots, \partial_{P}\left|\psi_{\boldsymbol{\omega}}\right\rangle\right\} \oplus \mathbb{C}^{P}, \\
& \text { subject to } 2 \operatorname{Re}\left(\left\langle x_{i}\left|\partial_{j}\right| \psi_{\boldsymbol{\omega}}\right\rangle\right)=\delta_{i j},\left\langle x_{i} \mid \psi_{\boldsymbol{\omega}}\right\rangle=0, \operatorname{Im}(V)=0,
\end{aligned}
$$

which we will call the Matsumoto bound. It was also shown in [49] that the bound is always saturable using individual measurements (which relaxes the requirement of collective measurements in the pure state case). However, there are no known efficient numerical algorithms to find the solutions $\left\{\left|x_{i}\right\rangle\right\}$ of the Mastumoto bound and it is not clear yet whether or not the Mastumoto bound will be useful in finding the optimal QEC protocols in our situation. In the following, we will start the proof of Theorem 2 by first sketching the proof of the Matsumoto bound and then reformulating it in such a way that the final optimization problem becomes an SDP, which will eventually be incorporated into the QEC protocol optimization procedure.

\subsection{Proof of Theorem 2}

\subsubsection{Proof and reformulation of the Matsumoto bound (Eq. (11))}

According to the Naimark's theorem [59], for any general measurement $\left\{M_{\ell}\right\}$ on $\mathcal{H}$ there exists a projective measurement $\left\{E_{\ell}\right\}$ on an extended space $\mathcal{H}_{M}$ (where $\mathcal{H} \subseteq \mathcal{H}_{M}$ ) satisfying $E_{\ell} E_{\ell^{\prime}}=\delta_{\ell \ell^{\prime}} E_{\ell}$ and $M_{\ell}=\Pi_{\mathcal{H}} E_{\ell} \Pi_{\mathcal{H}}$. We now define a set of vectors $\left|x_{i}\right\rangle \in \mathcal{H}_{M}$ :

$$
\left|x_{i}\right\rangle=\sum_{\ell}\left(\tilde{\omega}_{i}(\ell)-\omega_{i}\right) E_{\ell}\left|\psi_{\boldsymbol{\omega}}\right\rangle .
$$

One may see that, thanks to the projective nature of measurements $\left\{E_{\ell}\right\}$, scalar products of vectors $\left|x_{i}\right\rangle$ yield the covariance matrix of the estimator:

$$
V_{i j}=\left\langle x_{i} \mid x_{j}\right\rangle=\sum_{\ell, \ell^{\prime}}\left\langle\psi_{\boldsymbol{\omega}}\left|\left(\tilde{\omega}_{i}(\ell)-\omega_{i}\right) E_{\ell} E_{\ell^{\prime}}\left(\tilde{\omega}_{j}\left(\ell^{\prime}\right)-\omega_{j}\right)\right| \psi_{\boldsymbol{\omega}}\right\rangle=\Sigma_{i j}
$$

Now, instead of minimizing over the measurement $\left\{M_{\ell}\right\}$ on $\mathcal{H}$, we can perform the minimization directly over the vectors $\left|x_{i}\right\rangle \in \mathcal{H}_{M}$, imposing the following constraints:

$$
\operatorname{Im}\left(\left\langle x_{i} \mid x_{j}\right\rangle\right)=0, \quad\left\langle x_{i} \mid \psi_{\boldsymbol{\omega}}\right\rangle=0, \quad 2 \operatorname{Re}\left(\left\langle x_{i}\left|\partial_{j}\right| \psi_{\boldsymbol{\omega}}\right\rangle\right)=\delta_{i j} .
$$

These constraints correspond respectively to the projective nature of the measurement $\left\{E_{\ell}\right\}$ and the unbiasedness conditions as given in Eq. (4). At this point one may wonder how big the space $\mathcal{H}_{M}$ should be (as for a general measurement it might be arbitrary large). However, we can always map $\operatorname{span}\left\{\left|\psi_{\boldsymbol{\omega}}\right\rangle,\left\{\partial_{i}\left|\psi_{\boldsymbol{\omega}}\right\rangle,\left|x_{i}\right\rangle\right\}_{i=1}^{P}\right\} \subseteq \mathcal{H}_{M}$ isometrically to a $(2 P+1)$-dimensional space. Therefore when looking for the bound, under the constraint Eq. (14), it is enough to perform the minimization over $\left|x_{i}\right\rangle \in \operatorname{span}\left\{\left|\psi_{\boldsymbol{\omega}}\right\rangle, \partial_{1}\left|\psi_{\boldsymbol{\omega}}\right\rangle, \ldots, \partial_{P}\left|\psi_{\boldsymbol{\omega}}\right\rangle\right\} \oplus \mathbb{C}^{P}$, which results in equation Eq. (11). 
Finally, we show that indeed for any set of $\left|x_{i}\right\rangle$ satisfying Eq. (14) there exists a proper projective measurement on $\mathcal{H} \oplus \mathbb{C}^{P}$ and a locally unbiased estimator satisfying Eq. (12), and consequently there exists a corresponding general measurement on $\mathcal{H}$. To see this, notice that since $\forall_{i}\left\langle\psi_{\boldsymbol{\omega}} \mid x_{i}\right\rangle=0$ and $\forall_{i, j}\left\langle x_{i} \mid x_{j}\right\rangle \in \mathbb{R}$ one may choose an orthonormal basis $\left\{\left|b_{i}\right\rangle\right\}$ of $\operatorname{span}\left\{\left|\psi_{\boldsymbol{\omega}}\right\rangle,\left|x_{1}\right\rangle, \ldots,\left|x_{P}\right\rangle\right\}$ satisfying: $\forall_{i}\left\langle\psi_{\boldsymbol{\omega}} \mid b_{i}\right\rangle \in \mathbb{R} \backslash\{0\}$ and $\forall_{i, j}\left\langle x_{i} \mid b_{j}\right\rangle \in \mathbb{R}$. Then one can define a projective measurement:

$$
E_{\ell}=\left|b_{\ell}\right\rangle\left\langle b_{\ell}\left|(\ell=1, \ldots, P+1), \quad E_{0}=\mathbb{1}_{\operatorname{dim}\left(\mathcal{H}_{M}\right)}-\sum_{\ell=1}^{P+1}\right| b_{\ell}\right\rangle\left\langle b_{\ell}\right|
$$

with the corresponding estimator:

$$
\tilde{\omega}_{i}(\ell)=\frac{\left\langle b_{\ell} \mid x_{i}\right\rangle}{\left\langle b_{\ell} \mid \psi_{\boldsymbol{\omega}}\right\rangle}+\omega_{i}, \ell \geq 1, \quad \tilde{\omega}_{i}(0)=0
$$

which is locally unbiased and satisfies

$$
\left|x_{i}\right\rangle=\sum_{\ell=0}^{P+1}\left(\tilde{\omega}_{i}(\ell)-\omega_{i}\right) E_{\ell}\left|\psi_{\boldsymbol{\omega}}\right\rangle
$$

This proves Eq. (11).

Specifically, if $\operatorname{dim}(\mathcal{H}) \geq 2 P+1$ we may choose $\operatorname{span}\left\{\left|\psi_{\boldsymbol{\omega}}\right\rangle, \partial_{1}\left|\psi_{\boldsymbol{\omega}}\right\rangle, \ldots, \partial_{P}\left|\psi_{\boldsymbol{\omega}}\right\rangle\right\} \oplus \mathbb{C}^{P}$ as a subspace of $\mathcal{H}$ and optimize over $\left|x_{i}\right\rangle \in \mathcal{H}$. In this case we may also reformulate the Matsumoto bound in a slightly different form. First, note that any vectors $\left\{\left|x_{i}\right\rangle\right\}$ satisfying Eq. (14) need to be linearly independent. Let $\left\{\left|c_{i}\right\rangle\right\}_{i=1}^{P}$ be an orthonormal basis of $\operatorname{span}\left\{\left|x_{1}\right\rangle, \ldots,\left|x_{P}\right\rangle\right\}$, satisfying $\forall_{i, j} \operatorname{Im}\left\langle x_{i} \mid c_{j}\right\rangle=0$ (such a set may be generated using the Gram-Schmidt orthonormalization procedure). The locally unbiased conditions may now be rewritten as:

$$
2 \operatorname{Re}\left(\left\langle x_{i}\left|\partial_{j}\right| \psi_{\boldsymbol{\omega}}\right\rangle\right)=\sum_{k=1}^{P} 2 \operatorname{Re}\left(\left\langle x_{i} \mid c_{k}\right\rangle\left\langle c_{k}\left|\partial_{j}\right| \psi_{\boldsymbol{\omega}}\right\rangle\right)=\sum_{k=1}^{P}\left\langle x_{i} \mid c_{k}\right\rangle 2 \operatorname{Re}\left(\left\langle c_{k}\left|\partial_{j}\right| \psi_{\boldsymbol{\omega}}\right\rangle\right)=\delta_{i j},
$$

which (after introducing matrices $\mathcal{X}_{k i}=\left\langle c_{k} \mid x_{i}\right\rangle, D_{k j}=2 \operatorname{Re}\left(\left\langle c_{k}\left|\partial_{j}\right| \psi_{\boldsymbol{\omega}}\right\rangle\right)$ is equivalent to the matrix equality $\mathcal{X}^{T} D=\mathbb{1}_{P}$. From $\mathcal{X}^{T} D=\mathbb{1}_{P}$ we have $\mathcal{X}^{T}=D^{-1} \Rightarrow \operatorname{Tr}(W \cdot V)=\operatorname{Tr}\left(W \cdot \mathcal{X}^{T} \mathcal{X}\right)=$ $\operatorname{Tr}\left(W \cdot\left(D^{T} D\right)^{-1}\right)$, which gives

$$
\begin{aligned}
& \left.\min _{\left|c_{1}\right\rangle, \ldots,\left|c_{P}\right\rangle \in \mathcal{H}} \operatorname{Tr}\left(W \cdot\left(D^{T} D\right)^{-1}\right)\right), \\
& \text { where } D_{i j}=2 \operatorname{Re}\left\langle c_{i}\left|\partial_{j}\right| \psi_{\boldsymbol{\omega}}\right\rangle, \quad \text { subject to }\left\langle c_{i} \mid c_{j}\right\rangle=\delta_{i j} .
\end{aligned}
$$

This formulation will be more convenient to use when we will formulate the QEC protocol optimization problem as an SDP.

\subsubsection{Optimizing the error-correction codes}

Now we apply the reformulated Matsumoto bound to our task of identification of the optimal JNTQEC. Consider a given input state $\left|\psi_{\text {in }}\right\rangle$. Let $\mathcal{H}_{\mathcal{C}}$ be any code subspace of $\mathcal{H}_{S} \otimes \mathcal{H}_{A}$ containing $\left|\psi_{\text {in }}\right\rangle$ and satisfying the QEC conditions Eq. (6) - in order to be in accordance with the reformulated Matsumoto bound, this space may be required to be at least $2 P+1$ dimensional, but as we show in the following it will always be possible to reduce its dimensionality to $P+1$ effectively. Using QEC, our goal is to preserve an effective unitary evolution in the encoded space and coherently acquire the sensing signal. Therefore, we are effectively dealing with pure state $\left|\psi_{\boldsymbol{\omega}}\right\rangle$, which allows us to utilize Eq. (19) as a formula for the minimal cost of sensing multiple parameters.

The effective evolution after implementing QEC is given by

$$
\left|\psi_{\boldsymbol{\omega}}\right\rangle=\exp \left(-i T \sum_{j=1}^{P} \omega_{j} \Pi_{\mathcal{H}_{\mathcal{C}}}\left(G_{j} \otimes \mathbb{1}_{\operatorname{dim}\left(\mathcal{H}_{A}\right)}\right) \Pi_{\mathcal{H}_{\mathcal{C}}}\right)\left|\psi_{\text {in }}\right\rangle
$$

We focus on the estimation around point $\boldsymbol{\omega}=[0, \ldots, 0]$ (which can always be achieved by applying inverse Hamiltonian dynamics [52]) and denote $\left|c_{0}\right\rangle=\left|\psi_{\boldsymbol{\omega}=0}\right\rangle$ for notational simplicity. Then for 
any $\left|c_{i}\right\rangle \in \mathcal{H}_{\mathcal{C}}$ we have $2 \operatorname{Re}\left\langle c_{i}\left|\partial_{j}\right| \psi_{\boldsymbol{\omega}=0}\right\rangle=2 T \operatorname{Im}\left\langle c_{i}\left|\left(G_{j} \otimes \mathbb{1}_{\operatorname{dim}\left(\mathcal{H}_{A}\right)}\right)\right| c_{0}\right\rangle$, and according to Eq. (19) the minimum achievable cost for a fixed code space $\mathcal{H}_{\mathcal{C}}$ is given by:

$$
\begin{aligned}
& \left.\min _{\left|c_{1}\right\rangle, \ldots,\left|c_{P}\right\rangle \in \mathcal{H}_{\mathcal{C}}} \operatorname{Tr}\left(W \cdot\left(D^{T} D\right)^{-1}\right)\right), \\
& \text { where } D_{i j}=2 T \operatorname{Im}\left[\left\langle c_{i}\left|\left(G_{j} \otimes \mathbb{1}_{\operatorname{dim}\left(\mathcal{H}_{A}\right)}\right)\right| \psi_{\boldsymbol{\omega}}\right\rangle\right], \quad \text { subject to }\left\langle c_{i} \mid c_{j}\right\rangle=\delta_{i j} .
\end{aligned}
$$

From the above formulation it is clear that we may always reduce the code space $\mathcal{H}_{\mathcal{C}}$ to $\operatorname{span}\left\{\left|c_{k}\right\rangle\right\}_{k=0}^{P}$ without increasing the cost. Hence, the problem of optimization over both probes and errorcorrection protocols is now equivalent to identification of the set $\left\{\left|c_{k}\right\rangle\right\}_{k=0}^{P}$ that minimizes the cost with the constraint that $\mathcal{H}_{\mathcal{C}}=\operatorname{span}\left\{\left|c_{k}\right\rangle\right\}_{k=0}^{P}$ satisfies the QEC conditions.

To solve this problem, it will be convenient to formally extend the Hilbert space $\mathcal{H}_{S} \otimes \mathcal{H}_{A}$ by tensoring it with a $(P+1)$-dimensional reference space $\mathcal{H}_{\mathcal{R}}=\operatorname{span}\{|0\rangle, \ldots,|P\rangle\}$ (see Fig. 2c). This reference space will be representing the effective evolution of the probe state that happens within the code space and it will allow us to encode QEC conditions in a compact and numerically friendly way.

First, we introduce a matrix $Q \in \mathcal{L}\left(\mathcal{H}_{\mathcal{R}} \otimes \mathcal{H}_{S}\right)$ that represents a code

$$
Q=\operatorname{Tr}_{\mathcal{H}_{A}}\left(\left[\begin{array}{c}
\left|c_{0}\right\rangle \\
\vdots \\
\left|c_{P}\right\rangle
\end{array}\right]\left[\begin{array}{lll}
\left\langle c_{0}\right| & \cdots & \left\langle c_{P}\right|
\end{array}\right]\right)
$$

which, more formally, will be written as $Q=\operatorname{Tr}_{\mathcal{H}_{A}}\left(\sum_{k, l=0}^{P}|k\rangle\left\langle\left. l\right|_{\mathcal{H}_{\mathcal{R}}} \otimes \mid c_{k}\right\rangle\left\langle\left. c_{l}\right|_{\mathcal{H}_{S} \otimes \mathcal{H}_{A}}\right)\right.$. This matrix is proportional to the reduced density matrix of the maximum entangled state between $\mathcal{H}_{\mathcal{R}}$ and $\mathcal{H}_{\mathcal{C}}$. By its construction $Q \geq 0$ and contains all relevant information on the code states in $\mathcal{H}_{\mathcal{C}}$.

Next, we introduce effective generators $G_{i}^{\mathcal{R}}$ acting on $\mathcal{H}_{\mathcal{R}}$ so that they represent properly the action of the physical generators on the code space $\left[G_{i}^{\mathcal{R}}\right]_{k l}=\left\langle c_{k}\left|G_{i} \otimes \mathbb{1}_{\operatorname{dim}\left(\mathcal{H}_{A}\right)}\right| c_{l}\right\rangle$. The effective evolution generators are related with the $Q$ matrix via:

$$
\left(G_{i}^{\mathcal{R}}\right)^{T}=\operatorname{Tr}_{\mathcal{H}_{S}}\left[Q\left(\mathbb{1}_{P+1} \otimes G_{i}\right)\right] \quad i=1, \ldots, P .
$$

Note that the identity operator here acts on the reference space $\mathcal{H}_{\mathcal{R}}$, and not on the ancillary space $\mathcal{H}_{A}$. Taking into account the orthonormality of $\left|c_{k}\right\rangle$ and the QEC condition Eq. (6), we obtain the following constraints on $Q$

$$
\operatorname{Tr}_{\mathcal{H}_{S}}(Q)=\mathbb{1}_{P+1}, \forall_{S_{i} \in \mathcal{S}} \operatorname{Tr}_{\mathcal{H}_{S}}\left[Q\left(\mathbb{1}_{P+1} \otimes S_{i}\right)\right] \propto \mathbb{1}_{P+1}
$$

Let $\mathbb{1}_{d} / \sqrt{d},\left\{G_{i}\right\}_{i=1}^{P},\left\{S_{i}\right\}_{i=P+1}^{P^{\prime}},\left\{R_{i}\right\}_{i=P^{\prime}+1}^{d^{2}-1}$ form an orthonormal basis of Hermitian operators in $\mathcal{L}\left(\mathcal{H}_{S}\right)$ such that $\mathcal{S}=\operatorname{span}_{\mathbb{R}}\left\{\mathbb{1}_{d},\left(S_{i}\right)_{i=P+1}^{P^{\prime}}\right\}$. Any non-negative $Q$ satisfying Eqs. (23)-(24) has the following form:

$$
Q=\mathbb{1}_{P+1} \otimes \frac{\mathbb{1}_{d}}{d}+\sum_{i=1}^{P}\left(G_{i}^{\mathcal{R}}\right)^{T} \otimes G_{i}+\sum_{i=P+1}^{P^{\prime}} \nu_{i} \mathbb{1}_{P+1} \otimes S_{i}+\sum_{i=P^{\prime}+1}^{d^{2}-1} B_{i} \otimes R_{i} \geq 0
$$

where $\nu_{i} \in \mathbb{R}$ and $B_{i}$ are Hermitian. Conversely, for any nonnegative defined $Q \geq 0$, we can consider its purification $|Q\rangle \in \mathcal{H}_{\mathcal{R}} \otimes \mathcal{H}_{S} \otimes \mathcal{H}_{A}$, which when written as $|Q\rangle=\sum_{k=0}^{P}|k\rangle_{\mathcal{H}_{\mathcal{R}}} \otimes\left|c_{k}\right\rangle_{\mathcal{H}_{S} \otimes \mathcal{H}_{A}}$ yields the code states $\left|c_{k}\right\rangle$. Note that it implies that the rank of $Q$ corresponding to the dimension of the ancillary space. It is always sufficient to assume the dimension of the ancillary space to be $\operatorname{dim} \mathcal{H}_{A}=(P+1) d$. Therefore $\left\{G_{i}^{\mathcal{R}}\right\}$ is an achievable set of effective generators in $\mathcal{L}\left(\mathcal{H}_{\mathcal{R}}\right)$ (satisfying the QEC condition) if and only if there exist such $\nu_{i} \in \mathbb{R}$ and $B_{i}$, for which $Q \geq 0$.

Finally, in order to have an explicit dependence of the cost on the total time parameter $T$, we introduce a matrix $\Gamma=\frac{1}{2 T} D$, i.e. $\Gamma_{i j}=\operatorname{Im}\left[\left\langle c_{i}\left|G_{j} \otimes \mathbb{1}_{\operatorname{dim}\left(\mathcal{H}_{A}\right)}\right| c_{0}\right\rangle\right]=\operatorname{Im}\left[G_{j}^{\mathcal{R}}\right]_{i 0}$, and we end up with:

$$
\begin{aligned}
& \frac{1}{4 T^{2}} \min _{G_{i}^{\mathcal{R}}, B_{i}, \nu_{i}} \operatorname{Tr}\left(W\left(\Gamma^{T} \Gamma\right)^{-1}\right), \quad \text { where } \Gamma_{i j}=\operatorname{Im}\left[G_{j}^{\mathcal{R}}\right]_{i 0}, \\
& \text { subject to } \mathbb{1}_{P+1} \otimes \frac{\mathbb{1}_{d}}{d}+\sum_{i=1}^{P}\left(G_{i}^{\mathcal{R}}\right)^{T} \otimes G_{i}+\sum_{i=P+1}^{P^{\prime}} \nu_{i} \mathbb{1}_{P+1} \otimes S_{i}+\sum_{i=P^{\prime}+1}^{d^{2}-1} B_{i} \otimes R_{i} \geq 0 .
\end{aligned}
$$




\subsubsection{Reduction to an SDP}

In order to reformulate Eq. (26) as an SDP, we first show that we may assume without loss of generality that $\Gamma \sqrt{W^{-1}} \geq 0$. Note that for any full rank matrix $\Gamma$, the polar decomposition theorem implies that there always exists an orthonormal matrix $O$ such that $O \Gamma \sqrt{W^{-1}} \geq 0$. Next, as $\Gamma_{i j}=\operatorname{Im}\left[\left\langle i\left|G_{j}^{\mathcal{R}}\right| 0\right\rangle\right]$, multiplication $\Gamma$ by $O$ is equivalent to rotating the base in the reference space $\mathcal{H}_{\mathcal{R}}$. Since, according to Eq. (22) such a rotation cannot change the non-negativity of $Q$ and at the same time it does not affect the figure of merit $\operatorname{Tr}\left(W\left(\Gamma^{T} \Gamma\right)^{-1}\right)$, the statement is proven. To put Eq. (26) in the form of an SDP, we introduce a positive matrix $K \in \mathbb{R}^{P \times P}$ and a positive real number $w$. Now, using the following two relations,

$$
\begin{gathered}
{\left[\begin{array}{cc}
K & \mathbb{1}_{P} \\
\mathbb{1}_{P} & \Gamma \sqrt{W^{-1}}
\end{array}\right] \geq 0 \quad \Leftrightarrow \quad K \geq\left(\Gamma \sqrt{W^{-1}}\right)^{-1},} \\
{\left[\begin{array}{cc}
w \mathbb{1}_{P} & K \\
K & \mathbb{1}_{P}
\end{array}\right] \geq 0 \quad \Leftrightarrow \quad w \mathbb{1}_{P} \geq K^{2},}
\end{gathered}
$$

we see that $P \min w=\min \operatorname{Tr}\left(K^{2}\right)=\min \operatorname{Tr}\left(W\left(\Gamma^{T} \Gamma\right)^{-1}\right)$ in Eq. (8), making it equivalent to Eq. (26). Hence the problem takes the form of an SDP.

\subsection{Discussion}

It should be remarked that JNT-QEC do not contain SEP-QEC as a subclass. In SEP-QEC, unlike in JNT-QEC, the noises are not fully corrected in the entire space, and the decoherence is only avoided by choosing a properly mixed state input. In general one could combine both these approaches in a unified framework by dividing the set of all parameters into smaller subsets and then applying JNT-QEC for each of these subset separately - in this approach the SEP-QEC case would correspond to the situation where JNT-QEC optimization is applied to single parameter subsets. Such an optimization is in principle doable, but will involve much large numerical effort and it is not clear that it will lead to better protocols.

It is also worth noting that, apart from the improved metrological performance provided by QEC protocols when dealing with noisy systems, the above algorithm is also applicable in the noiseless scenario when $\mathcal{S}=\operatorname{span}_{\mathbb{R}}\left\{\mathbb{1}_{d}\right\}$. In such a situation no QEC is required (for simplicity we may still use $\mathcal{H}_{\mathcal{C}}$ for $\operatorname{span}\left\{\left|c_{k}\right\rangle\right\}_{k=0}^{P}$, but no recovery operation or projection $\Pi_{\mathcal{H}_{\mathcal{C}}}$ is needed during evolution), but the condition $\boldsymbol{\omega}=[0, \ldots, 0]$ (which is achievable by applying inverse Hamiltonian dynamics [52]) is still required, as otherwise the derivatives of the state may not scale linearly with $T$. In such situations, the solution of JNT-QEC yields an optimally ancilla-assisted sensing protcol under arbitrary system dynamics (Hamiltonitans) that resolves the potential incompatibility issues between sensing of different parameters. It should be stressed that our approach is universal and unlike existing approaches $[43,52,53]$ does not assume any specific structures of the Hamiltonians.

\section{Advantages of JNT-QEC over SEP-QEC}

In this section we investigate the potential advantages JNT-QEC provide compared with SEPQEC. The characteristic feature of the SEP-QEC is the division resources so that each part of the resources is used to measure a given parameter independently of the others. This is reflected in the form of the input probe state $\rho_{\text {in }}=\frac{1}{P} \sum_{i=1}^{P}\left|\psi_{i}\right\rangle\left\langle\psi_{i}\right|$. As a consequence we effectively measure each parameter only once in every $P$ repetitions of an experiment (corresponding to the $1 / P$ factor in the $\left.\rho_{\text {in }}\right)$. Therefore for a fixed total number of measurements, the uncertainty of estimating a given parameter will grow proportionally to $P$. Contrastingly, in JNT-QEC there is a chance to avoid the division of resources and use a single pure state and a single measurement to estimate all of them simultaneously. If there existed a code space, a state and a measurement which were all simultaneously optimal for all parameters, then the decrease the cost by a factor $P$ would be achievable. This would be the largest possible advantage offered by JNT-QEC. In general we can write (see Appx. A for more formal derivation):

$$
\Delta_{W}^{2} \tilde{\boldsymbol{\omega}}_{\mathrm{JNT}} \geq \frac{1}{P} \Delta_{W}^{2} \tilde{\boldsymbol{\omega}}_{\mathrm{SEP}}
$$


This inequality may be saturated only in special examples.

It is important to note that Theorem 2 gives us a recipe for identification of the optimal JNTQEC, while the explicit construction of SEP-QEC discussed in the proof of Theorem 1 was only aimed at demonstrating the possibility of the HS and hence the protocol was not optimized. Therefore, to make a fair comparison between the two approaches, we need to compare the performance of the best JNT-QEC with the best SEP-QEC.

Below we present a way to find the optimal SEP-QEC, i.e. the protocol for which all parameters are measured independently on mutually orthogonal subspaces. We will also provide a useful lower bound for the minimal cost achievable in such protocols.

\subsection{Optimization of SEP-QEC}

Adapting the results from [36] (see the section named "Geometrical picture"), we can infer that for a given set of $\left\{G_{i}\right\}$, the minimal variance achievable in estimation of a single parameter $\omega_{i}$ in SEP-QEC is given by:

$$
\Delta^{2} \tilde{\omega}_{i}=\frac{1}{F_{i}}, \quad F_{i}=4 T^{2} \underset{\widetilde{G}_{i \|} \in \mathcal{S} \oplus \min _{\mathbb{R}}\left\{G_{j}\right\}_{j \neq i}}{ }\left\|G_{i}-\widetilde{G}_{i \|}\right\|,
$$

where $\|\cdot\|$ denotes operator norm. Let $\left|\psi_{i}\right\rangle$ be the optimal state for measuring $\omega_{i}$. Therefore, using $\rho_{\text {in }}=\sum_{i=1}^{P} p_{i}\left|\psi_{i}\right\rangle\left\langle\psi_{i}\right|$ (where in the naive approach we would set all $p_{i}=\frac{1}{P}$ ) leads to the total cost $\Delta_{W}^{2} \tilde{\boldsymbol{\omega}}_{\mathrm{SEP}}=\sum_{i=1}^{P} \frac{1}{p_{i}} \frac{W_{i i}}{F_{i}}$. After optimization over $p_{i}$ is performed (keeping in mind that $\left.\sum_{i=1}^{P} p_{i}=1, p_{i} \geq 0\right)$ we get $\Delta_{W}^{2} \tilde{\boldsymbol{\omega}}_{\mathrm{SEP}}=\left(\sum_{i=1}^{P} \sqrt{\frac{W_{i i}}{F_{i}}}\right)^{2}$.

Next, we may still improve the peromance of SEP-QEC by choosing a different QEC code based on a new set of generators obtained via a linear transformation on the parameters $A \in \mathbb{R}^{P \times P}$ :

$$
\boldsymbol{\omega}^{\prime}=\boldsymbol{\omega} A^{-1}, \quad \boldsymbol{G}^{\prime}=A \boldsymbol{G}, \quad W^{\prime}=A W A^{T},
$$

so that the cost function remains unchanged.

Note also, that rescaling any generator by constant factor has no impact on the results, so we may restrict to linear transformations satisfying $\left(A A^{T}\right)_{i i}=1$. Therefore the cost for the optimal SEP-QEC is given by:

$$
\begin{aligned}
\Delta_{W}^{2} \tilde{\boldsymbol{\omega}}_{\mathrm{SEP}} & =\min _{A: \forall_{i}\left(A A^{T}\right)_{i i}=1}\left(\sum_{i=1}^{P} \sqrt{\frac{\left(A W A^{T}\right)_{i i}}{F_{i}(A)}}\right)^{2}, \\
\text { where } F_{i}(A)=4 T^{2} & \min _{\widetilde{G}_{i \|} \in \mathcal{S} \oplus \operatorname{mpan}_{\mathbb{R}}\left\{(A \boldsymbol{G})_{j}\right\}_{j \neq i}}\left\|(A \boldsymbol{G})_{i}-\widetilde{G}_{i \|}\right\| .
\end{aligned}
$$

We want to stress that introducing this (relatively complicated) procedure of SEP-QEC optimization is necessary in order to distniguish the cases where the true advantage is offered by the joint multi-parameter approach compared with the situation where the advatage is only apparent and results from a suboptimal choice of separate protocols.

Formula Eq. (32) is rather complicated and hard to compute in general. However, in case $W=\mathbb{1}$ the following lower bound is valid:

$$
\Delta_{W}^{2} \tilde{\boldsymbol{\omega}}_{\mathrm{SEP}} \geq \min _{a_{i}: \sum a_{i}^{2}=1} \frac{P^{2}}{T^{2}\left(\lambda_{+}^{*}-\lambda_{-}^{*}\right)^{2}}, \quad G^{*}=\sum_{i=1}^{P} a_{i} G_{i},
$$

where $\lambda_{ \pm}^{*}$ are extreme eigenvalues of $G^{*}$.

Since the reasoning leading to Eq. (29) holds for any set of generators, not necessary the ones optimal for SEP-QEC, therefore, the optimal joint-estimation cost, in case $W=\mathbb{1}$, may be bounded as (see Appx. A for a formal derivation):

$$
\Delta_{W}^{2} \tilde{\boldsymbol{\omega}}_{\mathrm{JNT}} \geq \min _{i} \frac{P}{T^{2}\left(\lambda_{i+}-\lambda_{i-}\right)^{2}}, \quad \text { for any set of } G_{i}
$$


where $\lambda_{i \pm}$ are extreme eigenvalues of $G_{i}$.

In order to provide the reader with some intuition on the concept presented above, we discuss below two extreme cases illustrating the apparent and maximal advantage of joint-estimation protocols over separate ones. These examples deal with noiseless scenarios where JNT-QEC is always optimal and are aimed to prepare the reader for more physical noisy examples discussed in Sec. 6. Still, in order not to introduce additional abbreviations we will still use the acronyms JNT-QEC, SEP-QEC to describe separate and joint estimation schemes, even though the role of $\mathrm{QEC}$ in these examples is trivial.

\subsection{Example: Apparent advantage of JNT-QEC}

Consider a noiseless physical system comprising $P=2^{r}$ qubits (for technical reasons we require the number of qubits to be the integer power of 2). Each qubit, regarded as a spin $1 / 2$, senses the $z$-component of a local magnetic field which is assumed to be independent for different qubit locations. The corresponding sensing Hamiltonian for this system reads:

$$
H=\sum_{i=1}^{P} \omega_{i} G_{i}, \quad G_{i}=\sigma_{z}^{(i)}=\mathbb{1}^{\otimes(i-1)} \otimes \sigma_{z} \otimes \mathbb{1}^{\otimes(P-i)}
$$

and the cost matrix is assumed to be $W=\mathbb{1}$. The minimum variance of estimating each parameter independently is lower bounded by $\Delta^{2} \tilde{\omega}_{i} \geq \frac{1}{T^{2}\left(\lambda_{+}-\lambda_{-}\right)^{2}}$. As the maximum and the minimum eigenvalues of each $G_{i}$ are $\lambda_{i \pm}= \pm 1$, the minimal variance reads $\Delta^{2} \tilde{\omega}_{i}=\frac{1}{4 T^{2}}$. According to Eq. (34)) this implies that the optimal JNT-QEC cost is lower bounded by $\frac{P}{4 T^{2}}$. Moreover, since the state $|\psi\rangle=\frac{1}{\sqrt{2^{P}}}(|+\rangle+|-\rangle)^{\otimes P}$ is simultaneously optimal for all the parameters (and the tensor structure guarantees no measurement incompatibility issue), this bound is saturable and hence

$$
\Delta_{W}^{2} \tilde{\boldsymbol{\omega}}_{\mathrm{JNT}}=\frac{P}{4 T^{2}} .
$$

On the other hand, the estimation strategy where each parameter $\omega_{i}$ is estimated separately leads to the cost equal $\frac{P^{2}}{4 T^{2}}$. Therefore, one may naively think that it is an example of superiority JNT-QEC over SEP-QEC.

However, the separate protocol may be significantly improved here, by estimating different combinations of the parameters. According to Eq. (33), we should be looking for a proper linear combination of $G_{i}$ with the biggest difference of extreme eigenvalues. The most obvious choice is $G^{*}=\frac{1}{\sqrt{P}} \sum_{i=1}^{P} G_{i}$, for which $\lambda_{ \pm}^{*}=\sqrt{P}$, and therefore $\Delta_{W}^{2} \tilde{\boldsymbol{\omega}}_{\mathrm{SEP}} \geq \frac{P}{4 T^{2}}$, which is exactly equal to $\Delta_{W}^{2} \tilde{\boldsymbol{\omega}}_{\mathrm{JNT}}$ ! Below we show, that this bound may be saturated.

Let the matrix $A$ defining the transformation to the new set of parameters be proportional to a Hadamard matrix of size $P \times P$ (i.e. square matrix whose entries are either +1 or -1 and whose rows are mutually orthogonal) defined as follows:

$$
A_{i j}=\frac{1}{\sqrt{P}} \prod_{k=0}^{r-1}(-1)^{i_{k} j_{k}},
$$

where the indices of the matrix are written using the binary representation, $i=\sum_{k=0}^{r-1} i_{k} 2^{k}$, where $i_{k}$ represent the binary digit of $i$ at position $k$. Then for $\boldsymbol{G}^{\prime}=A \boldsymbol{G}$ we have:

$$
\lambda_{i \pm}^{\prime}= \pm \sqrt{P}, \quad\left|\lambda_{i \pm}^{\prime}\right\rangle=\bigotimes_{j=1}^{P}\left| \pm A_{i j}\right\rangle, \quad \forall_{j \neq i}\left\langle\lambda_{i \pm}^{\prime}\left|G_{j}^{\prime}\right| \lambda_{i \pm}^{\prime}\right\rangle=\left\langle\lambda_{i \pm}^{\prime}\left|G_{j}^{\prime}\right| \lambda_{i \mp}^{\prime}\right\rangle=0 .
$$

As a result every $\omega_{i}^{\prime}$ may be measured separately on subspace $\operatorname{span}\left\{\left|\lambda_{i+}^{\prime}\right\rangle,\left|\lambda_{i-}^{\prime}\right\rangle\right\}$ with precision $\frac{1}{4 P T^{2}}$. Therefore, for the initial sate $\rho_{\text {in }}=\frac{1}{P} \sum_{i=1}^{P}\left|\psi_{i}\right\rangle\left\langle\psi_{i}\right|$ with $\left|\psi_{i}\right\rangle=\frac{1}{\sqrt{2}}\left(\left|\lambda_{i+}\right\rangle+\left|\lambda_{i-}\right\rangle\right)$ we get

$$
\Delta_{W}^{2} \tilde{\boldsymbol{\omega}}_{\mathrm{SEP}}=\frac{P}{4 T^{2}},
$$

which is exactly the same as the optimal $\Delta_{W}^{2} \tilde{\boldsymbol{\omega}}_{\mathrm{JNT}}$. We see that the apparent advantage of a JNT-QEC disappears once a proper combinations of parameters are estimated in the SEP-QEC. 


\subsection{Example: Maximal advantage of JNT-QEC}

As a contrasting example, here we discuss a situation where the advantage of JNT-QEC is genuine and is the maximal possible. This example also provides an intuition, what relation between the generators $G_{i}$ is responsible for this.

Consider a noiseless system $\mathcal{H}_{S}=\operatorname{span}\{|i\rangle\}_{i=0}^{P}$ with Hamiltonian:

$$
H=\sum_{i=1}^{P} \omega_{i} G_{i}, \quad G_{i}=\frac{1}{\sqrt{2}}(|0\rangle\langle i|+| i\rangle\langle 0|) .
$$

We focus on the estimation around point $\boldsymbol{\omega}=[0, \ldots, 0]$ with the cost matrix $W=\mathbb{1}$. As all generators are orthonormal $\operatorname{Tr}\left(G_{i} G_{j}\right)=\delta_{i j}$, the difference between extreme eigenvalues of any normalized combination of them, such as $G^{*}$ in Eq. (33), is at most equal to $\sqrt{2}$. Therefore, the optimal SEP-QEC cost is bounded by

$$
\Delta_{W}^{2} \tilde{\boldsymbol{\omega}}_{\mathrm{SEP}} \geq \frac{P^{2}}{2 T^{2}}
$$

Importantly, the state $\left|\psi_{\text {in }}\right\rangle=|0\rangle$ is simultaneously optimal for measuring all the parameters. There is also no measurement incompatibility problem as the optimal measurement for all the parameters is the measurement in the basis $|i\rangle$. Therefore,

$$
\Delta_{W}^{2} \tilde{\boldsymbol{\omega}}_{\mathrm{JNT}}=\frac{P}{2 T^{2}}
$$

is achievable, and hence the there is a factor $P$ decrease in the cost in case of JNT-QEC compared with the optimal SEP-QEC protocols.

\section{Examples}

Here we provide representative examples of a large class of multi-parameter estimation models, where there are unavoidable tradeoffs in determining the optimal states and measurements that arise due to the multi-parameter nature of the problem. Our methods are useful in identifying optimal strategies in all such models, provided the structure of noise admits the achievability of the HS via application of the most general QEC schemes. By the construction of our algorithm (Theorem 2), we have the guarantee that the solutions found are the optimal ones. For an interested reader, a broader discussion and generalizations of the results presented in this section, including proofs and analytical constructions of the codes are provided in Appx. B, Appx. C, Appx. D.

\subsection{Single qubit case}

Consider first the simplest single-qubit case with $d=2$. The HS is achievable via QEC only in the case of single-rank Pauli noise (specified by a single Hermitian Lindbladian $L$ ) [34]. Without loss of generality we can set $L=\sigma_{z}$ (the Pauli-Z matrix). Since $\mathcal{S}=\operatorname{span}\left\{\mathbb{1}, \sigma_{z}\right\}$, at most two parameters may be estimated in a qubit system with the HS (as $\operatorname{dim}\left(\mathcal{S}^{\perp}\right)=2$ ). However, it turns out that when the multi-parameter HNLS condition is met there is no benefit in performing the more sophisticated JNT-QEC compared to SEP-QEC, which is shown analytically in Appx. B.

\subsection{Two qubits in a magnetic field}

In order to appreciate the superiority of JNT-QEC over SEP-QEC, let us consider a two-qubit model which is a multi-parameter generalization of the one from [37]. Consider two localized qubits, coupled to a magnetic field, which is constant in both time and space, apart from some small fluctuations in the $z$ direction. These fluctuations are assumed to be uncorrelated in time, but maximally anticorrelated in space (for the two qubits they have always opposite signs). Such a system may be effectively described by Eq. (1) with $H=\frac{1}{2} \sum_{i=1}^{2} \boldsymbol{\omega} \cdot \boldsymbol{\sigma}^{(i)}$ (where $\boldsymbol{\sigma}^{(i)}=\left[\sigma_{x}^{(i)}, \sigma_{y}^{(i)}, \sigma_{z}^{(i)}\right]$ acts on the $i^{\text {th }}$ atom) and a single Lindblad operator $L=\sqrt{2 \gamma}\left(\sigma_{z}^{(1)}-\sigma_{z}^{(2)}\right)$. It can be shown 


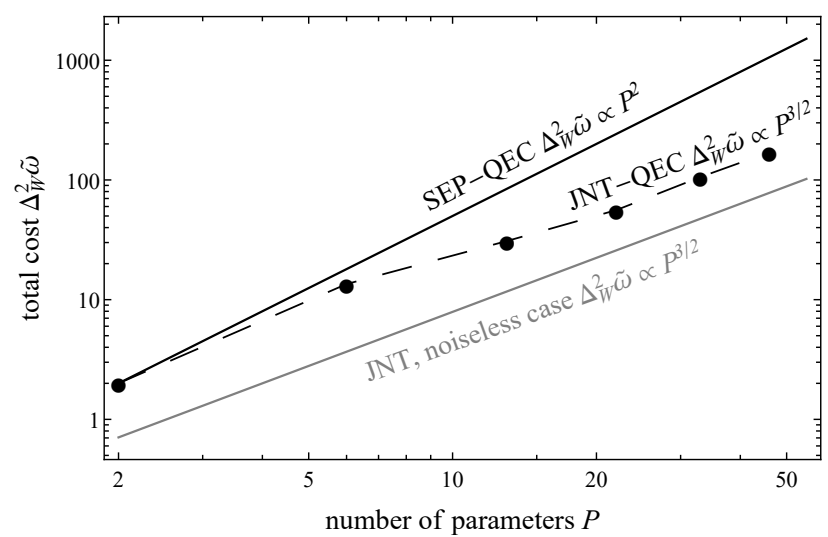

Figure 3: Numerical results for the optimal JNT-QEC strategy for estimating the $S U(d)$ generators under the noise $J_{z}$ (black points joined by dashed line), in contrast with the optimal precision asymptotically achievable by SEP-QEC $\Delta_{W}^{2} \tilde{\boldsymbol{\omega}}=\frac{P^{2}}{2 T^{2}}$ (black solid line) and the lower bound $\Delta_{W}^{2} \tilde{\boldsymbol{\omega}}=\frac{P^{3 / 2}}{4 T^{2}}$ asymptotically achievable only in the noiseless case (solid gray line).

that the minimal cost when each parameter is estimated with the optimal individual parameter strategy is $\Delta^{2} \tilde{\omega}_{x, y, z}=\frac{1}{4 T^{2}}$. At the same time, in accordance with the discussion presented in Sec. 5, the best precision achievable using SEP-QEC for the standard cost matrix $W=\mathbb{1}$, is $\Delta_{W}^{2} \tilde{\boldsymbol{\omega}}_{\mathrm{SEP}}=\frac{P^{2}}{4 T^{2}}=\frac{9}{4 T^{2}}$ - see Appx. C for the formal derivation of the above formulas.

Below we present the result of numerical optimization of the JNT-QEC approach (found by the algorithm presented in Theorem 2 and reconstructed to its analytical form). We will use the standard Bell states notation:

$$
\begin{array}{ll}
\left|\Phi_{+}\right\rangle=\frac{1}{\sqrt{2}}(|\uparrow \uparrow\rangle+|\downarrow \downarrow\rangle), & \left|\Phi_{-}\right\rangle=\frac{1}{\sqrt{2}}(|\uparrow \uparrow\rangle-|\downarrow \downarrow\rangle), \\
\left|\Psi_{+}\right\rangle=\frac{1}{\sqrt{2}}(|\uparrow \downarrow\rangle+|\downarrow \uparrow\rangle), \quad\left|\Psi_{-}\right\rangle=\frac{1}{\sqrt{2}}(|\uparrow \downarrow\rangle-|\downarrow \uparrow\rangle) .
\end{array}
$$

Entanglement with ancilla will be abbreviated in the subscript $|\psi\rangle \otimes|i\rangle_{A} \equiv|\psi\rangle_{i}$. Using the numerical algorithm we have found out, that the optimal code space has the form

$$
\begin{aligned}
\left|c_{0}\right\rangle & =-\cos (\varphi)\left|\Phi_{+}\right\rangle_{1}+\frac{i}{\sqrt{2}} \sin (\varphi)\left(\left|\Phi_{+}\right\rangle_{2}+\left|\Phi_{-}\right\rangle_{3}\right), \\
\left|c_{1}\right\rangle & =-i \sin (\varphi)\left|\Phi_{+}\right\rangle_{1}-\cos (\varphi)\left|\Psi_{+}\right\rangle_{2}, \\
\left|c_{2}\right\rangle & =-\sin (\varphi)\left|\Phi_{-}\right\rangle_{1}-i \cos (\varphi)\left|\Psi_{+}\right\rangle_{3}, \\
\left|c_{3}\right\rangle & =-\frac{1}{\sqrt{2}} \sin (\varphi)\left(\left|\Phi_{-}\right\rangle_{2}+\left|\Phi_{+}\right\rangle_{3}\right)+\cos (\varphi)\left|\Psi_{+}\right\rangle_{4},
\end{aligned}
$$

where the input state is $\left|\psi_{\boldsymbol{\omega}=\mathbf{0}}\right\rangle=\left|c_{0}\right\rangle$. Note that the presence of the last term in $\left|c_{3}\right\rangle$ (entangled with $|4\rangle_{A}$ ) is necessary to satisfy the QEC conditions. The value of $\varphi$ can be found analytically and the minimal total cost of estimation $\Delta_{W}^{2} \tilde{\boldsymbol{\omega}}$ is achieved for:

$$
\cos (\varphi)=\sqrt{\frac{\sqrt{7+4 \sqrt{2}-3}}{4 \sqrt{2}-2}} \approx 0.39,
$$

while the corresponding optimal cost is:

$$
\Delta_{W}^{2} \tilde{\boldsymbol{\omega}}_{\mathrm{JNT}} \approx \frac{5.31}{4 T^{2}}
$$

As we see a significant improvement has been achieved here compared to SEP-QEC. 


\section{3 $S U(d)$ generators' estimation}

Finally let us consider an example of estimating parameters associated with $S U(d)$ generators which shows an asymptotic advantage (with the number of parameters) of the JNT-QEC over the SEP-QEC protocol. Let $\mathcal{H}_{S}$ be $d$-dimensional Hilbert space, which for a more intuitive notation may be regarded as the one associated with a spin- $j$ particle (where $d=2 j+1$ ).

First, let us recall the noiseless case, where the Hamiltonian $H=\sum_{i=1}^{d^{2}-1} \omega_{i} G_{i}$ is composed of all $P=d^{2}-1 S U(d)$ generators. The generators together with the identity $\left\{\frac{1}{\sqrt{d}} \mathbb{1}, G_{1}, \ldots, G_{d^{2}-1}\right\}$ form an orthonormal basis of Hermitian operators on $\mathcal{H}_{S}$. We focus on the estimation around point $\boldsymbol{\omega}=[0, \ldots, 0]$ with the cost matrix $W=\mathbb{1}$. Since all $G_{i}$ are orthonormal the maximum spread between their maximum and minimal eigenvalues as well as any normalized combination of them is $\sqrt{2}$. Using the same line of reasoning as presented in Sec. 5.3 we conclude that

$$
\Delta_{W}^{2} \tilde{\boldsymbol{\omega}}_{\mathrm{SEP}} \geq \frac{P^{2}}{2 T^{2}}=\frac{\left(d^{2}-1\right)^{2}}{2 T^{2}} .
$$

The bound for $\Delta_{W}^{2} \tilde{\boldsymbol{\omega}}_{\text {JNT }}$ may be derived analytically. To achieve that, we use the following chain of inequalities involving the QFI matrix:

$$
\sum_{i=1}^{d^{2}-1} \Delta^{2} \tilde{\omega}_{i} \geq \sum_{i=1}^{d^{2}-1}\left(F^{-1}\right)_{i i} \geq \sum_{i=1}^{d^{2}-1} \frac{1}{F_{i i}} \geq \frac{\left(d^{2}-1\right)^{2}}{\sum_{i=1}^{d^{2}-1} F_{i i}},
$$

where the first one is the CR inequality and the rest are general algebraic properties of positive semidefinite matrices. What remains to be done is to derive a proper bound for the trace of the QFI matrix. For any input state $\left|\psi_{\text {in }}\right\rangle \in \mathcal{H}_{S} \otimes \mathcal{H}_{A}$ we have:

$$
F_{i i}=4 T^{2}\left(\left\langle\psi_{\boldsymbol{\omega}}\left|G_{i}^{2} \otimes \mathbb{1}\right| \psi_{\boldsymbol{\omega}}\right\rangle-\left\langle\psi_{\boldsymbol{\omega}}\left|G_{i} \otimes \mathbb{1}\right| \psi_{\boldsymbol{\omega}}\right\rangle^{2}\right) \leq 4 T^{2}\left\langle\psi_{\boldsymbol{\omega}}\left|G_{i}^{2} \otimes \mathbb{1}\right| \psi_{\boldsymbol{\omega}}\right\rangle .
$$

Taking into account the normalization of $G_{i}$ and noting that $\sum_{i=1}^{d^{2}-1} G_{i}^{2}$ is the Casimir operator of the $S U(d)$ algebra, and as such is proportional to the identity, we get that $\sum_{i=1}^{d^{2}-1} G_{i}^{2}=\frac{d^{2}-1}{d} \mathbb{1}$. Therefore:

$$
\sum_{i=1}^{d^{2}-1} F_{i i} \leq 4 T^{2}\left\langle\psi_{\boldsymbol{\omega}}\left|\sum_{i=1}^{d^{2}-1} G_{i}^{2} \otimes \mathbb{1}\right| \psi_{\boldsymbol{\omega}}\right\rangle=4 T^{2} \frac{d^{2}-1}{d} .
$$

After substituting the above to Eq. (48) we get

$$
\Delta_{W}^{2} \tilde{\boldsymbol{\omega}}_{\mathrm{JNT}}=\sum_{i=1}^{d^{2}-1} \Delta^{2} \tilde{\omega}_{i} \geq \frac{d\left(d^{2}-1\right)}{4 T^{2}} \approx \frac{P^{3 / 2}}{4 T^{2}} .
$$

The example of a state which saturates the above bound is $\left|\psi_{\text {in }}\right\rangle=\frac{1}{\sqrt{d}} \sum_{k=1}^{d}|k\rangle_{S} \otimes|k\rangle_{A}[52,53]$. For such a state the QFI matrix is given by $F_{i j}=\delta_{i j} \frac{4 T^{2}}{d}$, so the second and the third inequalities in Eq. (48) become equalities. As $\operatorname{Im}\left(\left\langle\psi_{\boldsymbol{\omega}}\left|\Lambda_{i} \Lambda_{j}\right| \psi_{\boldsymbol{\omega}}\right\rangle\right) \propto\left\langle\psi_{\boldsymbol{\omega}}\left|\left[G_{i} \otimes \mathbb{1}, G_{j} \otimes \mathbb{1}\right]\right| \psi_{\boldsymbol{\omega}}\right\rangle=0$, the first one (the CR bound) is saturable as well. Note that the role of ancillae here is to make optimal measurements with respect to different parameters compatible. As a result, we see that optimal $\Delta_{W}^{2} \tilde{\boldsymbol{\omega}}_{\text {JNT }}$ is approximately $2 \sqrt{P}$ times smaller than $\Delta_{W}^{2} \tilde{\boldsymbol{\omega}}_{\text {SEP }}$.

Let us now consider a noisy version with a single Lindblad operator $J_{z}=\sum_{k=-j}^{j} k|k\rangle\langle k|$. From Theorem 1 we know that only parameters associated with generators $G_{i} \notin \operatorname{span}_{\mathbb{R}}\left\{\mathbb{1}, J_{z}, J_{z}^{2}\right\}$ may be estimated with the HS. Therefore we consider the Hamiltonian $H=\sum_{i=1}^{P} \omega_{i} G_{i}$ composed of $P=d^{2}-3$ of $S U(d)$ generators orthogonal to $\left.\operatorname{span}_{\mathbb{R}}\left\{\mathbb{1}, J_{z}, J_{z}^{2}\right\}\right)$, with the standard cost matrix $W=\mathbb{1}$ (such cost makes the problem independent on choosing peculiar set of $\left\{G_{i}\right\}$ ). In Fig. 3, we present numerical results for such a problem, and we observe a significant advantage over the SEPQEC protocol as well as strong indication of the asymptotic $P^{3 / 2}$ scaling identical to the noiseless case. Even though the optimal JNT-QEC code cannot be written down analytically in a concise way, in Appx. D we provide an analytical suboptimal construction achieving the $P^{3 / 2}$ scaling, supporting the numerical results. The scaling advantage we prove here is not trivial because there are no decoherence-free subspaces in the system. 


\section{Conclusions and outlook}

We have generalized previous results on single-parameter error-corrected metrology to the multiparameter scheme, obtaining a necessary and sufficient condition (HNLS) for the achievability of the HS. In case of scenarios when HNLS is satisfied, we developed an efficient numerical algorithm (formulated as an SDP) to find the optimal QEC protocol, including the optimal input state, QEC codes and measurements. Our algorithm is applicable to arbitrary system dynamics as long as the HS is achievable (including noiseless cases), which contrasts previous works where special dynamics of quantum system is assumed $[43,52,53]$ or the estimation is performed on fixed quantum states [66]. However, it still remains open in which situations the requirement of noiseless ancillae could be removed [37,38] and whether QEC is still helpful in the case when HNLS is violated, as in the single-parameter case [41, 74].

We also remark that our way of formulating the Knill-Laflamme conditions [72] as a positive semidefinite constraint Eq. (25) is novel and may have applications beyond error-corrected quantum metrology.

Finally, we note that in this paper we have followed the frequentist estimation approach, and in principle more stringent HS bounds might be derived when following the Bayesian approach as was demonstrated recently in the single parameter case [75].

\section{Acknowledgements}

We thank Mengzhen Zhang, John Preskill and Francesco Albarelli for inspiring discussions and Joseph Renes for his input in formulating the final form of the SDP. WG and RDD acknowledge support from the National Science Center (Poland) grant No. 2016/22/E/ST2/00559. SZ and LJ acknowledge support from the ARL-CDQI (W911NF-15-2-0067, W911NF-18-2-0237), ARO (W911NF-18-1-0020, W911NF-18-1-0212), ARO MURI (W911NF-16-1-0349), AFOSR MURI (FA955014-1-0052, FA9550-15-1-0015), DOE (de-sc0019406), NSF (EFMA-1640959), and the Packard Foundation (2013-39273). 


\section{A The proof of $\Delta_{W}^{2} \tilde{\boldsymbol{\omega}}_{\mathrm{JNT}} \geq \frac{1}{P} \Delta_{W}^{2} \tilde{\boldsymbol{\omega}}_{\mathrm{SEP}}$}

In the reasoning presented in the beginning of Sec. 5 we have assumed that, when estimating a single parameter, the minimal variance of the estimator $\Delta^{2} \tilde{\omega}_{i}$ achievable in SEP-QEC is no bigger than the one achievable in JNT-QEC (even if we focus only on this particular parameter). This statement is not so obvious as both protocols impose different constraints on the code space:

$$
\begin{aligned}
& \text { JNT-QEC : } \forall_{j} \Pi_{\mathcal{H}_{C}}\left(G_{j} \otimes \mathbb{1}\right) \Pi_{\mathcal{H}_{C}} \not \Pi_{\mathcal{H}_{C}}, \\
& \text { SEP-QEC : } \forall_{j \neq i} \Pi_{\mathcal{H}_{C_{i}}}\left(G_{j} \otimes \mathbb{1}\right) \Pi_{\mathcal{H}_{C_{i}}} \propto \Pi_{\mathcal{H}_{C_{i}}}, \quad \Pi_{\mathcal{H}_{C_{i}}}\left(G_{i} \otimes \mathbb{1}\right) \Pi_{\mathcal{H}_{C_{i}}} \not \Pi_{\mathcal{H}_{C_{i}}},
\end{aligned}
$$

where none of them is weaker or stronger than the other one.

Therefore, here we show directly that having JNT-QEC with accuracy $\Delta_{W}^{2} \tilde{\boldsymbol{\omega}}_{\text {JNT }}$, one may always construct SEP-QEC giving $\Delta_{W}^{2} \tilde{\boldsymbol{\omega}}_{\mathrm{SEP}}=P \cdot \Delta_{W}^{2} \tilde{\boldsymbol{\omega}}_{\mathrm{JNT}}$. Let $\mathcal{H}_{\mathcal{C}}$ be the code space used in JNT-QEC. Without loss of generality let us assume that $W$ is diagonal (otherwise we apply a transformation of parameters that diagonalizes $W$ ) and $\operatorname{dim}\left(\mathcal{H}_{\mathcal{C}}\right) \geq 2 P+1$ (otherwise we trivially extend it using an additional ancilla). From the Matsumoto bound (see Eq. (11)) the optimal cost is given by:

$$
\begin{aligned}
& \Delta_{W}^{2} \tilde{\boldsymbol{\omega}}_{\mathrm{JNT}}=\min _{\left\{\left|x_{i}\right\rangle\right\}} \operatorname{Tr}(W V), \quad \text { where } V_{i j}=\left\langle x_{i} \mid x_{j}\right\rangle, \\
& \text { for }\left|x_{i}\right\rangle \in \mathcal{H}_{\mathcal{C}}, \\
& \text { subject to } 2 \operatorname{Re}\left(\left\langle x_{i}\left|\partial_{j}\right| \psi_{\boldsymbol{\omega}}\right\rangle\right)=\delta_{i j},\left\langle x_{i} \mid \psi_{\boldsymbol{\omega}}\right\rangle=0, \operatorname{Im}(V)=0,
\end{aligned}
$$

where for $\omega=0$ we have $\left.\partial_{j}\left|\psi_{\boldsymbol{\omega}}\right\rangle\right|_{\omega=0}=-i T G_{j}^{\mathcal{C}}\left|\psi_{\boldsymbol{\omega}=\mathbf{0}}\right\rangle$. We define $\mathcal{H}_{\mathcal{C}_{i}}=\operatorname{span}_{\mathbb{R}}\left\{\left|c_{0}^{i}\right\rangle,\left|c_{1}^{i}\right\rangle\right\}$ with:

$$
\left|c_{0}^{i}\right\rangle=\frac{1}{\sqrt{2}}\left(\left|\psi_{\boldsymbol{\omega}=\mathbf{0}}\right\rangle|0\rangle_{A_{i}}+\frac{1}{\sqrt{\left\langle x_{i} \mid x_{i}\right\rangle}}\left|x_{i}\right\rangle|1\rangle_{A_{i}}\right), \quad\left|c_{1}^{i}\right\rangle=\frac{1}{\sqrt{2}}\left(\frac{1}{\sqrt{\left\langle x_{i} \mid x_{i}\right\rangle}}\left|x_{i}\right\rangle|0\rangle_{A_{i}}+\left|\psi_{\boldsymbol{\omega}=\mathbf{0}}\right\rangle|1\rangle_{A_{i}}\right)
$$

where $\left|x_{i}\right\rangle$ are the result of optimization Eq. (53). As $\left|\psi_{\boldsymbol{\omega}=\mathbf{0}}\right\rangle,\left|x_{i}\right\rangle \in \mathcal{H}_{\mathcal{C}}$, obviously the noise acts trivially on $\mathcal{H}_{\mathcal{C}_{i}}$. Moreover:

$$
\begin{aligned}
\left\langle c_{0}^{i}\left|G_{j} \otimes \mathbb{1}\right| c_{1}^{i}\right\rangle & =\frac{1}{2 \sqrt{\left\langle x_{i} \mid x_{i}\right\rangle}}\left(\left\langle\psi_{\boldsymbol{\omega}=\mathbf{0}}\left|G_{j}^{\mathcal{C}}\right| x_{i}\right\rangle+\left\langle x_{i}\left|G_{j}^{\mathcal{C}}\right| \psi_{\boldsymbol{\omega}=\mathbf{0}}\right\rangle\right)=\frac{1}{T \sqrt{\left\langle x_{i} \mid x_{i}\right\rangle}} \delta_{i j} \\
\left\langle c_{0}^{i}\left|G_{j} \otimes \mathbb{1}\right| c_{0}^{i}\right\rangle & =\frac{1}{2}\left(\left\langle\psi_{\boldsymbol{\omega}=\mathbf{0}}\left|G_{j}^{\mathcal{C}}\right| \psi_{\boldsymbol{\omega}=\mathbf{0}}\right\rangle+\frac{1}{\left\langle x_{i} \mid x_{i}\right\rangle}\left\langle x_{i}\left|G_{j}^{\mathcal{C}}\right| x_{i}\right\rangle\right)=\left\langle c_{1}^{i}\left|G_{j} \otimes \mathbb{1}\right| c_{1}^{i}\right\rangle,
\end{aligned}
$$

so also condition $\forall_{j \neq i} \Pi_{\mathcal{H}_{\mathcal{C}_{i}}} G_{j} \Pi_{\mathcal{H}_{\mathcal{C}_{i}}} \propto \Pi_{\mathcal{H}_{\mathcal{C}_{i}}}$ is satisfied.

Therefore SEP-QEC with initial state $\rho_{\text {in }}=\frac{1}{P} \sum_{i=1}^{P}\left|c_{0}^{i}\right\rangle\left\langle c_{0}^{i}\right|$ and $\mathcal{H}_{\mathcal{C}_{i}}$ defined above leads to the total cost $\Delta_{W}^{2} \tilde{\boldsymbol{\omega}}_{\mathrm{SEP}}=\sum_{i=1}^{P} P \cdot W_{i i}\left\langle x_{i} \mid x_{i}\right\rangle=P \cdot \Delta_{W}^{2} \tilde{\boldsymbol{\omega}}_{\mathrm{JNT}}$. From this reasoning we see clearly that the largest possible advantage of JNT-QEC over SEP-QEC is to decreasing the total cost by a factor $P$.

\section{B Optimality of SEP-QEC in the single-qubit model}

Let us consider the most general case of a two-parameter quantum estimation problem under Markovian noise in a 2-dimensional Hilbert space when the HS achievability condition is satisfied. Without loss of generality we assume that the Lindblad operator is $L=\sigma_{z}$, the Hamiltonian is $H=\omega_{x} \sigma_{x}+\omega_{y} \sigma_{y}$ and the cost matrix is diagonal:

$$
\left[\begin{array}{cc}
W_{x x} & 0 \\
0 & W_{y y}
\end{array}\right]
$$

(otherwise, one can always apply a proper transformation in the parameter space $\boldsymbol{\omega}^{\prime}=\boldsymbol{\omega} A^{-1}$ which diagonalizes the cost matrix, without changing orthonormality of the generators). Below we show that for such a problem there is no advantage of JNT-QEC over SEP-QEC.

First let us consider the optimal SEP-QEC. Each generator has eigenvalues $\left|\lambda_{x / y \pm}\right\rangle= \pm 1$ and each parameter may be estimated with precision $\Delta^{2} \tilde{\omega}_{x / y}=\frac{1}{4 T^{2}}$ [34]. Moreover, as the cost is 
diagonal, there is no point in applying an additional transformation in the optimization procedure given in Eq. (32) - indeed, any matrix $A$ satisfying $\forall_{i}\left(A A^{T}\right)_{i i}=1$ preserves the eigenvalues of the generators as well as $\operatorname{Tr}\left(A W A^{T}\right)$. Therefore the optimal SEP-QEC cost is simply given by $\Delta_{W}^{2} \tilde{\boldsymbol{\omega}}_{\mathrm{SEP}}=\frac{\left(\sqrt{W_{x x}}+\sqrt{W_{y y}}\right)^{2}}{4 T^{2}}$. We will show below that this cannot be outperformed by the optimal JNT-QECs.

For the sake of notation simplicity, when tensoring an operator acting on $\mathcal{H}_{S}$ with the identity on $\mathcal{H}_{A}$, the part $\otimes \mathbb{1}$ will be omitted and we will denote $\sigma_{i} \otimes \mathbb{1}$ simply as $\sigma_{i}$ (unless this leads to ambiguity).

First, we note that the diagonal elements of the QFI matrix for the state $\left|\psi_{\boldsymbol{\omega}}\right\rangle$ are

$$
\begin{aligned}
F_{i i} & =4 T^{2}\left(\left\langle\psi_{\boldsymbol{\omega}}\left|\sigma_{i} \Pi_{\mathcal{H}_{\mathcal{C}}} \sigma_{i}\right| \psi_{\boldsymbol{\omega}}\right\rangle-\left|\left\langle\psi_{\boldsymbol{\omega}}\left|\Pi_{\mathcal{H}_{\mathcal{C}}} \sigma_{i}\right| \psi_{\boldsymbol{\omega}}\right\rangle\right|^{2}\right) \\
& \leq 4 T^{2}\left\langle\psi_{\boldsymbol{\omega}}\left|\sigma_{i} \Pi_{\mathcal{H}_{\mathcal{C}}} \sigma_{i}\right| \psi_{\boldsymbol{\omega}}\right\rangle \quad(i=x, y) .
\end{aligned}
$$

Moreover

$$
\begin{aligned}
\Delta_{W}^{2} \tilde{\boldsymbol{\omega}} & =W_{x x} \Delta^{2} \tilde{\omega}_{x}+W_{y y} \Delta^{2} \tilde{\omega}_{y} \geq \frac{W_{x x}}{F_{x x}}+\frac{W_{y y}}{F_{y y}}=\frac{1}{F_{x x}+F_{y y}}\left(\frac{W_{x x}}{\frac{F_{x x}}{F_{x x}+F_{y y}}}+\frac{W_{y y}}{\frac{F_{y y}}{F_{x x}+F_{y y}}}\right) \\
& \geq \frac{1}{F_{x x}+F_{y y}} \min _{p \in[0,1]}\left(\frac{W_{x x}}{p}+\frac{W_{y y}}{1-p}\right)=\frac{\left(\sqrt{W_{x x}}+\sqrt{W_{y y}}\right)^{2}}{F_{x x}+F_{y y}} .
\end{aligned}
$$

Therefore we may focus on an upper bound for $\sum_{i=x, y}\left\langle\psi_{\boldsymbol{\omega}}\left|\sigma_{i} \Pi_{\mathcal{H}_{\mathcal{C}}} \sigma_{i}\right| \psi_{\boldsymbol{\omega}}\right\rangle$.

Let $\left\{\left|c_{0}\right\rangle,\left|c_{1}\right\rangle,\left|c_{2}\right\rangle\right\}$ be an orthonormal basis of $\mathcal{H}_{\mathcal{C}} \subseteq \mathcal{H}_{S} \otimes \mathcal{H}_{A}$. These vectors can be written down as

$$
\left|c_{i}\right\rangle=\cos \left(\varphi^{i}\right)|0\rangle\left|A_{0}^{i}\right\rangle+\sin \left(\varphi^{i}\right)|1\rangle\left|A_{1}^{i}\right\rangle,
$$

where $\left|A_{0 / 1}^{i}\right\rangle$ are normalized states in $\mathcal{H}_{A}$ and $\varphi^{i} \in\left[0, \frac{\pi}{2}\right]$ (a potential complex phase is incorporated in the definition of $\left.\left|A_{0 / 1}^{i}\right\rangle\right)$. The QEC condition requires $\forall_{i, j}\left\langle c_{i}\left|\sigma_{z}\right| c_{j}\right\rangle=\lambda \delta_{i j}$, which leads to the following two constraints: (i) $\forall_{i} \cos ^{2}\left(\varphi^{i}\right)-\sin ^{2}\left(\varphi^{i}\right)=\lambda$ means all $\varphi^{i}$ are equal (therefore superscript $i$ will be omitted); (ii) $\forall_{i \neq j} \cos ^{2}(\varphi)\left\langle A_{0}^{i} \mid A_{0}^{j}\right\rangle-\sin ^{2}(\varphi)\left\langle A_{1}^{i} \mid A_{1}^{j}\right\rangle=0$. Together with the orthonormality condition $\forall_{i \neq j} \cos ^{2}(\varphi)\left\langle A_{0}^{i} \mid A_{0}^{j}\right\rangle+\sin ^{2}(\varphi)\left\langle A_{1}^{i} \mid A_{1}^{j}\right\rangle=0$, we have

$$
\begin{gathered}
\left|c_{i}\right\rangle=\cos (\varphi)|0\rangle\left|A_{0}^{i}\right\rangle+\sin (\varphi)|1\rangle\left|A_{1}^{i}\right\rangle, \\
\forall_{i, j}\left\langle A_{0}^{i} \mid A_{0}^{j}\right\rangle=\delta_{i j}, \quad\left\langle A_{1}^{i} \mid A_{1}^{j}\right\rangle=\delta_{i j} .
\end{gathered}
$$

Note that there is no fixed relationship between sets $\left\{\left|A_{0}^{i}\right\rangle\right\}_{i=0}^{i=2}$ and $\left\{\left|A_{1}^{i}\right\rangle\right\}_{i=0}^{i=2}$-in particular it may happen that $\operatorname{span}\left\{\left|A_{0}^{i}\right\rangle\right\} \neq \operatorname{span}\left\{\left|A_{1}^{i}\right\rangle\right\}$. Effective generators in the chosen basis are given as:

$$
\begin{aligned}
& \left(G_{x}^{\mathcal{R}}\right)_{j i}=\left\langle c_{j}\left|\sigma_{x}\right| c_{i}\right\rangle=\frac{\sin (2 \varphi)}{2}\left(\left\langle A_{0}^{j} \mid A_{1}^{i}\right\rangle+\left\langle A_{1}^{j} \mid A_{0}^{i}\right\rangle\right), \\
& \left(G_{y}^{\mathcal{R}}\right)_{j i}=\left\langle c_{j}\left|\sigma_{y}\right| c_{i}\right\rangle=i \frac{\sin (2 \varphi)}{2}\left(\left\langle A_{1}^{j} \mid A_{0}^{i}\right\rangle-\left\langle A_{0}^{j} \mid A_{1}^{i}\right\rangle\right) .
\end{aligned}
$$

We focus on estimation around point $\boldsymbol{\omega}=[0, \ldots, 0]$ for which $\left|\psi_{\boldsymbol{\omega}}\right\rangle=\left|\psi_{\text {in }}\right\rangle=\left|c_{0}\right\rangle$. Then

$$
\begin{aligned}
\left\langle\psi_{\boldsymbol{\omega}}\left|\sigma_{x / y} \Pi_{\mathcal{H}_{\mathcal{C}}} \sigma_{x / y}\right| \psi_{\boldsymbol{\omega}}\right\rangle= & \sum_{i=0}^{2}\left\langle c_{0}\left|\sigma_{x / y}\right| c_{i}\right\rangle\left\langle c_{i}\left|\sigma_{x / y}\right| c_{0}\right\rangle= \\
& \frac{\sin ^{2}(2 \varphi)}{4}\left(\sum_{i=0}^{2}\left|\left\langle A_{0}^{0} \mid A_{1}^{i}\right\rangle\right|^{2}+\left|\left\langle A_{1}^{0} \mid A_{0}^{i}\right\rangle\right|^{2} \pm 2 \operatorname{Re}\left(\left\langle A_{1}^{0} \mid A_{0}^{i}\right\rangle\left\langle A_{1}^{i} \mid A_{0}^{0}\right\rangle\right)\right) .
\end{aligned}
$$

Since for each $k=0 / 1$, states $\left\{\left|A_{k}^{i}\right\rangle\right\}_{i=0}^{2}$ are mutually orthonormal,

$$
\sum_{i=x, y}\left\langle\psi\left|\sigma_{i} \Pi_{\mathcal{H}_{\mathcal{C}}} \sigma_{i}\right| \psi\right\rangle=\frac{\sin ^{2}(2 \varphi)}{2} \cdot \sum_{i=0}^{2}\left(\left|\left\langle A_{0}^{0} \mid A_{1}^{i}\right\rangle\right|^{2}+\left|\left\langle A_{1}^{0} \mid A_{0}^{i}\right\rangle\right|^{2}\right) \leq \sin ^{2}(2 \varphi) \leq 1,
$$

where the first inequality is saturated if and only if both $\left|A_{0}^{0}\right\rangle \in \operatorname{span}\left\{\left|A_{1}^{i}\right\rangle\right\}_{i=0}^{2}$ and $\left|A_{1}^{0}\right\rangle \in$ $\operatorname{span}\left\{\left|A_{0}^{i}\right\rangle\right\}_{i=0}^{2}$. Using Eq. (57) and Eq. (58) we get $\Delta_{W}^{2} \tilde{\boldsymbol{\omega}} \geq \frac{\left(\sqrt{W_{x x}}+\sqrt{W_{y y}}\right)^{2}}{4 T^{2}}$. This implies that the JNT-QEC stategy cannot outperform the best SEP-QEC strategy. 


\section{The optimal SEP protocol for sensing all magnetic field components in presence of correlated dephasing noise in the two-qubit model}

Here we prove formally, that the optimal precision achievable in the second example from the main text is $\Delta_{W}^{2} \tilde{\boldsymbol{\omega}}_{\mathrm{SEP}}=\frac{9}{4 T^{2}}$.

Let us briefly review the problem. We consider a two-atom system with Hamiltonian $H=$ $\frac{1}{2} \sum_{i=1}^{2} \boldsymbol{\omega} \cdot \boldsymbol{\sigma}^{(i)}$ (where $\boldsymbol{\sigma}^{(i)}=\left[\sigma_{x}^{(i)}, \sigma_{y}^{(i)}, \sigma_{z}^{(i)}\right]$ acts on the $i^{\text {th }}$ atom) and a single Lindblad operator $L=\sqrt{2 \gamma}\left(\sigma_{z}^{(1)}-\sigma_{z}^{(2)}\right)$, therefore

$$
\mathcal{S}=\operatorname{span}_{\mathbb{R}}\left\{\mathbb{1}, L, L^{2}\right\}=\operatorname{span}_{\mathbb{R}}\left\{\mathbb{1}, \sigma_{z}^{(1)}-\sigma_{z}^{(2)}, \sigma_{z}^{(1)} \sigma_{z}^{(2)}\right\} .
$$

As for all three generators the minimal and the maximal eigenvalues are respectively -1 and +1 , we immediately see that $\Delta^{2} \tilde{\omega}_{x, y, z} \geq \frac{1}{4 T^{2}}$ and from that $\Delta_{W}^{2} \tilde{\boldsymbol{\omega}}_{\mathrm{SEP}} \geq \frac{P^{2}}{4 T^{2}}=\frac{9}{4 T^{2}}$ (which cannot be improved by applying transformation $A$ ). Below we show that such a precision is indeed achievable.

First, $\omega_{z}$ can be estimated using a decoherence-free subspace [76] $\operatorname{span}\left\{\left|\psi_{z}\right\rangle, \frac{1}{2}\left(\sigma_{z}^{(1)}+\sigma_{z}^{(2)}\right)\left|\psi_{z}\right\rangle\right\}$, where $\left|\psi_{z}\right\rangle=\frac{1}{\sqrt{2}}(|\uparrow \uparrow\rangle+|\downarrow \downarrow\rangle)$, which leads to the precision $\Delta^{2} \omega_{z}=\frac{1}{4 T^{2}}$.

In case of $\omega_{x}$ the situation is slightly more complicated, as using the analogue approach the subspace $\operatorname{span}\left\{\left|\psi_{x}\right\rangle, \frac{1}{2}\left(\sigma_{x}^{(1)}+\sigma_{x}^{(2)}\right)\left|\psi_{x}\right\rangle\right\}$ would not satisfy the QEC conditions. To get the desired estimation precision we need to find the state $\left|\psi_{x}\right\rangle$ which is optimal for measuring $\omega_{x}$ (from the point of view of noiseless, single-parameter estimation) and for which

$$
\mathcal{H}_{\mathcal{C}_{x}}=\operatorname{span}\left\{\left|\psi_{x}\right\rangle, \frac{1}{2}\left(\sigma_{x}^{(1)}+\sigma_{x}^{(2)}\right)\left|\psi_{x}\right\rangle\right\}
$$

satisfies the QEC condition:

$$
\begin{aligned}
\Pi_{\mathcal{H}_{\mathcal{C}_{x}}}\left(\sigma_{z}^{(1)}-\sigma_{z}^{(2)}\right) \Pi_{\mathcal{H}_{\mathcal{C}_{x}}} & \propto \Pi_{\mathcal{H}_{\mathcal{C}_{x}}}, \\
\Pi_{\mathcal{H}_{\mathcal{C}_{x}}} \sigma_{z}^{(1)} \sigma_{z}^{(2)} \Pi_{\mathcal{H}_{\mathcal{C}_{x}}} & \propto \Pi_{\mathcal{H}_{\mathcal{C}_{x}}}
\end{aligned}
$$

and, moreover, other generators act trivially inside $\mathcal{H}_{\mathcal{C}_{x}}$ :

$$
\begin{aligned}
& \Pi_{\mathcal{H}_{\mathcal{C}_{y}}\left(\sigma_{y}^{(1)}+\sigma_{y}^{(2)}\right) \Pi_{\mathcal{H}_{\mathcal{C}_{x}}}} \propto \Pi_{\mathcal{H}_{\mathcal{C}_{x}}}, \\
& \Pi_{\mathcal{H}_{\mathcal{C}_{z}}\left(\sigma_{z}^{(1)}+\sigma_{z}^{(2)}\right) \Pi_{\mathcal{H}_{\mathcal{C}_{x}}}} \propto \Pi_{\mathcal{H}_{\mathcal{C}_{x}}} .
\end{aligned}
$$

It is known that for single-parameter frequency estimation the optimal state corresponds to an equally weighted superposition of states with minimal and maximal eigenvalues of the generator. For $\frac{1}{2}\left(\sigma_{x}^{(1)}+\sigma_{x}^{(2)}\right)$ it will be:

$$
\left|\psi_{x}^{\varphi}\right\rangle=\frac{1}{2 \sqrt{2}} \cdot\left((|\uparrow\rangle+|\downarrow\rangle)(|\uparrow\rangle+|\downarrow\rangle)+e^{i \varphi}(|\uparrow\rangle-|\downarrow\rangle)(|\uparrow\rangle-|\downarrow\rangle)\right)
$$

for any $\varphi \in \mathbb{R}$. Note, that any superposition of $\left|\psi_{x}^{\varphi}\right\rangle$ (with different $\varphi$ ) entangled with separated ancillae is still optimal for sensing $\omega_{x}$. Therefore, we can take $\left|\psi_{x}\right\rangle=\frac{1}{\sqrt{2}}\left(\left|\psi_{x}^{0}\right\rangle|1\rangle_{A}+\left|\psi_{x}^{\pi}\right\rangle|2\rangle_{A}\right)$. We have:

$$
\begin{gathered}
\left|\psi_{x}\right\rangle=\frac{1}{2}\left((|\uparrow \uparrow\rangle+|\downarrow \downarrow\rangle)|1\rangle_{A}+(|\uparrow \downarrow\rangle+|\downarrow \uparrow\rangle)|2\rangle_{A}\right), \\
\frac{1}{2}\left(\sigma_{x}^{(1)}+\sigma_{x}^{(2)}\right)\left|\psi_{x}\right\rangle=\frac{1}{2}\left((|\uparrow \downarrow\rangle+|\downarrow \uparrow\rangle)|1\rangle_{A}+(|\uparrow \uparrow\rangle+|\downarrow \downarrow\rangle)|2\rangle_{A}\right) .
\end{gathered}
$$

and then the code space Eq. (66) satisfies Eq. (67) and Eq. (68). It gives as $\Delta^{2} \omega_{x}=\frac{1}{4 T^{2}}$. Analogous reasoning could be provided for $\omega_{y}$.

Therefore for $\rho_{\text {in }}=\frac{1}{3} \sum_{i=x, y, z}\left|\psi_{i}\right\rangle\left\langle\psi_{i}\right|$ we have $\Delta_{W}^{2} \tilde{\boldsymbol{\omega}}=\frac{9}{4 T^{2}}$ in line with general considerations on the performance of the SEP-QEC codes as given in Sec. 5. 


\section{Estimating the $S U(d)$ generators}

Below we present an example of a JNT-QEC protocol allowing one to achieve the total cost $\Delta_{W}^{2} \tilde{\boldsymbol{\omega}}=\Theta\left(P^{\frac{3}{2}}\right)$ for the last example in the main text. For clarification, we treat $d$-dimensional Hilbert space as a single spin- $j$ particle $(d=2 j+1)$ and we use the notation where $\{|k\rangle\}_{k=-j}^{j}$ is the eigenbasis of the $J_{z}$ operator.

We consider a problem where the noise generator $J_{z}$ and the unitary evolution $H$ read:

$$
J_{z}=\sum_{k=-j}^{j} k|k\rangle\langle k|, \quad H=\sum_{i=1}^{P} \omega_{i} G_{i},
$$

where $G_{i}$ is an orthonormal basis of $\mathcal{S}^{\perp}$ - the orthogonal complement of $\mathcal{S}=\operatorname{span}\left\{\mathbb{1}, J_{z}, J_{z}^{2}\right\}$ (therefore $P=d^{2}-3$ ). For technical reasons we distinguish three groups of operators that form the basis $\left\{G_{i}\right\}$ :

- Real off-diagonal: $G_{k l}^{R}=\frac{1}{\sqrt{2}}(|k\rangle\langle l|+| l\rangle\langle k|)$

- Imaginary off-diagonal: $G_{k l}^{I}=\frac{i}{\sqrt{2}}(|k\rangle\langle l|-| l\rangle\langle k|)$

- Diagonal: $G_{i}^{D}=\sum_{k=-j}^{j} g_{i}^{k}|k\rangle\langle k|$

and in what follows we prove the scaling $\Theta\left(P^{\frac{3}{2}}\right)$ for each group. For simplicity, we assume, that $j$ is an integer (for half-integer $j$ the proof remains almost the same) and in this section we focus on the estimation around point $\boldsymbol{\omega}=[0, \ldots, 0]$ and set $T=1$.

Real off-diagonal generators. We take $\operatorname{dim}\left(\mathcal{H}_{A}\right)=\operatorname{dim}\left(\mathcal{H}_{S}\right)$ and the state $\left|\psi_{\boldsymbol{\omega}}\right\rangle=\left|\psi^{R}\right\rangle=$ $\frac{1}{\sqrt{2 j+1}} \sum_{k=-j}^{j}|k\rangle|k\rangle_{A} \in \mathcal{H}_{S} \otimes \mathcal{H}_{A}$, we have

$$
\left\langle\psi^{R}\left|J_{z}\right| \psi^{R}\right\rangle=0, \quad\left\langle\psi^{R}\left|J_{z}^{2}\right| \psi^{R}\right\rangle=\frac{j(j+1)}{3} .
$$

We construct the code space in the following way. First, we act on $|\psi\rangle$ with generators: $G_{k l}^{R}|\psi\rangle=$ $\frac{1}{\sqrt{2}}\left(|k\rangle|l\rangle_{A}+|l\rangle|k\rangle_{A}\right)$ and then we "fix it" to satisfy the QEC condition by extending ancilla $\mathcal{H}_{A} \rightarrow \mathcal{H}_{A} \oplus \mathcal{H}_{B}$ and adding more terms:

$$
\left|c_{k l}^{R}\right\rangle=\frac{p}{\sqrt{2}}\left(|k\rangle|l\rangle_{A}+|l\rangle|k\rangle_{A}\right)++q|j\rangle|k l j\rangle_{B}+r|-j\rangle|k l(-j)\rangle_{B}+s|0\rangle|k l 0\rangle_{B},
$$

where $\left\langle k l m \mid k^{\prime} l^{\prime} m^{\prime}\right\rangle_{B}=\delta_{(k l m)\left(k^{\prime} l^{\prime} m^{\prime}\right)}$. Then the QEC condition is equivalent to:

$$
\begin{aligned}
& \left\langle c_{k l}^{R} \mid c_{k l}^{R}\right\rangle=p^{2}+q^{2}+r^{2}+s^{2}=1, \\
& \left\langle c_{k l}^{R}\left|J_{z}\right| c_{k l}^{R}\right\rangle=\frac{p^{2}}{2}(k+l)+\left(q^{2}-r^{2}\right) j=0, \\
& \left\langle c_{k l}^{R}\left|J_{z}^{2}\right| c_{k l}^{R}\right\rangle=\frac{p^{2}}{2}\left(k^{2}+l^{2}\right)+\left(q^{2}+r^{2}\right) j^{2}=\frac{j(j+1)}{3} .
\end{aligned}
$$

The off-diagonal terms are automatically zero, no matter what $p, q, r, s$ are. We can write down $q^{2}, r^{2}$ and $s^{2}$ as linear functions of $p^{2}$ :

$$
\begin{aligned}
& q^{2}=\frac{1}{2 j^{2}}\left(\frac{j(j+1)}{3}-\frac{p^{2}}{2}\left(k^{2}+l^{2}+j(k+l)\right)\right), \\
& r^{2}=\frac{1}{2 j^{2}}\left(\frac{j(j+1)}{3}-\frac{p^{2}}{2}\left(k^{2}+l^{2}-j(k+l)\right)\right), \\
& s^{2}=1-p^{2}-\frac{1}{j^{2}}\left(\frac{j(j+1)}{3}-\frac{p^{2}}{2}\left(k^{2}+l^{2}\right)\right) .
\end{aligned}
$$

Note that $p$ is a valid coefficient if the above set of equations has a solution (i.e. if the right-hand sides are positive). As $-2 j \leq k+l \leq 2 j, k^{2}+l^{2} \leq 2 j^{2}$, this always holds provided $p^{2}=\frac{1}{6}$. For the code space $\mathcal{H}_{\mathcal{C}_{R}}$ spanned by vectors constructed in such a way, we have

$$
\left(G_{k l}^{R}\right)^{\mathcal{H}_{\mathcal{C}_{R}}}\left|\psi^{R}\right\rangle=\frac{p}{\sqrt{2 j+1}}\left|c_{k l}^{R}\right\rangle
$$


The QFIs are

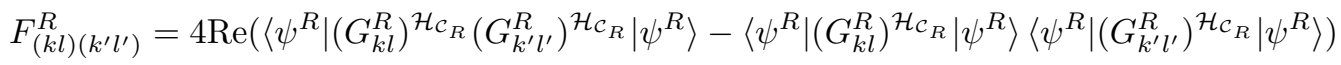

which in our case simplifies to:

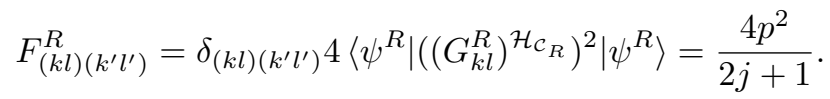

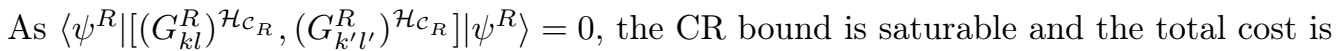

$$
\sum_{k>l} \Delta^{2} \omega_{k l}^{R}=\frac{2 j+1}{4} \sum_{k>l} \frac{1}{p^{2}}=\frac{3 j(2 j+1)^{2}}{2}=\Theta\left(P^{\frac{3}{2}}\right) .
$$

Imaginary off-diagonal generators. The reasoning is analogous to the previous case. Note that using different ancillary spaces for real and imaginary generators is needed. Even though $\left\langle\psi^{R}\left|G_{k l}^{R} J_{z} G_{k^{\prime} l^{\prime}}^{R}\right| \psi^{R}\right\rangle \propto \delta_{k l, k^{\prime} l^{\prime}}$ and $\left\langle\psi^{R}\left|G_{k l}^{I} J_{z} G_{k^{\prime} l^{\prime}}^{I}\right| \psi^{R}\right\rangle \propto \delta_{k l, k^{\prime} l^{\prime}}$ are satisfied automatically, $\left\langle\psi^{R}\left|G_{k l}^{R} J_{z} G_{k^{\prime} l^{\prime}}^{I}\right| \psi^{R}\right\rangle \propto \delta_{k l, k^{\prime} l^{\prime}}$ may not be true.

Diagonal generators. The number of diagonal generators scales like $\Theta(j)$ (whereas for offdiagonal elements the scaling is $\Theta\left(j^{2}\right)$ ), implying that the estimation with respect to diagonal generators does not contribute significantly to the overall scaling. Therefore we could simply use the SEP-QEC approach. Following [36], any traceless generator may by written down as:

$$
G_{i}^{D}=\frac{1}{2} \operatorname{Tr}\left(\left|G_{i}^{D}\right|\right)\left(\rho_{i+}-\rho_{i-}\right) .
$$

We define states $\left|c_{i+}\right\rangle,\left|c_{i-}\right\rangle$ as purifications of these density matrices by using mutually orthogonal ancillary subspaces $\mathcal{H}_{A i+}, \mathcal{H}_{A i-}$ :

$$
\rho_{i+/-}=\operatorname{Tr}_{A i+/-}\left(\left|c_{i+/-}\right\rangle\left\langle c_{i+/-}\right|\right)
$$

Therefore

$$
\begin{aligned}
\left\langle c_{i+}\left|G_{i}^{D}\right| c_{i+}\right\rangle & =\frac{1}{2} \operatorname{Tr}\left(\left|G_{i}^{D}\right|\right) \geq \frac{1}{2 \sqrt{2 j+1}}, \\
\left\langle c_{i-}\left|G_{i}^{D}\right| c_{i-}\right\rangle & =-\frac{1}{2} \operatorname{Tr}\left(\left|G_{i}^{D}\right|\right) \leq-\frac{1}{2 \sqrt{2 j+1}},
\end{aligned}
$$

and from that for code space $\operatorname{span}\left\{\left|c_{i+}\right\rangle,\left|c_{i-}\right\rangle\right\}$ and input state $\frac{1}{\sqrt{2}}\left(\left|c_{i+}\right\rangle+\left|c_{i-}\right\rangle\right)$ we have $F_{\omega_{i}} \geq$ $\frac{1}{2 j+1}$. For a single-parameter problem the CR bound is always saturable so using the SEP-QEC approach for the input state

$$
\rho_{\text {in }}^{D}=\frac{1}{2 j-2} \sum_{i=1}^{2 j-2} \frac{1}{2}\left(\left|c_{i+}\right\rangle+\left|c_{i-}\right\rangle\right)\left(\left\langle c_{i+}\right|+\left\langle c_{i-}\right|\right)
$$

we have

$$
\sum_{i=1}^{2 j-2} \Delta^{2} \omega_{i}^{D}=(2 j-2) \sum_{i=1}^{2 j-2} \frac{1}{F_{\omega_{i}}} \leq(2 j+1)(2 j-2)^{2}=\Theta\left(P^{\frac{3}{2}}\right) .
$$

Results. Finally, combining all above, for the input state:

$$
\rho_{\text {in }}=\frac{1}{3}\left(\left|\psi^{R}\right\rangle\left\langle\psi^{R}|+| \psi^{I}\right\rangle\left\langle\psi^{I}\right|+\rho^{D}\right)
$$

(with properly applied QEC protocol) we get

$$
\sum_{i=1}^{P} \Delta^{2} \omega_{i}=\Theta\left(P^{\frac{3}{2}}\right)
$$




\section{References}

[1] V. Giovannetti, S. Lloyd, and L. Maccone, Quantum metrology, Phys. Rev. Lett. 96, 010401 (2006).

[2] M. G. A. Paris, Quantum estimation for quantum technologies, Int. J. Quantum Inf. 07, 125 (2009).

[3] V. Giovannetti, S. Lloyd, and L. Maccone, Advances in quantum metrology, Nat. Photonics 5, 222 (2011).

[4] G. Toth and I. Apellaniz, Quantum metrology from a quantum information science perspective, J. Phys. A: Math. Theor. 47, 424006 (2014).

[5] R. Demkowicz-Dobrzański, M. Jarzyna, and J. Kołodyński, in Prog. Optics, Vol. 60, edited by E. Wolf (Elsevier, 2015) pp. 345-435.

[6] R. Schnabel, Squeezed states of light and their applications in laser interferometers, Phys. Rep. 684, 1 (2017).

[7] C. L. Degen, F. Reinhard, and P. Cappellaro, Quantum sensing, Rev. Mod. Phys. 89, 035002 (2017).

[8] L. Pezzè, A. Smerzi, M. K. Oberthaler, R. Schmied, and P. Treutlein, Quantum metrology with nonclassical states of atomic ensembles, Rev. Mod. Phys. 90, 035005 (2018).

[9] S. Pirandola, B. R. Bardhan, T. Gehring, C. Weedbrook, and S. Lloyd, Advances in photonic quantum sensing, Nat. Photonics 12, 724 (2018).

[10] C. M. Caves, Quantum-mechanical noise in an interferometer, Phys. Rev. D 23, 1693 (1981).

[11] M. Holland and K. Burnett, Interferometric detection of optical phase shifts at the heisenberg limit, Phys. Rev. Lett. 71, 1355 (1993).

[12] H. Lee, P. Kok, and J. P. Dowling, A quantum rosetta stone for interferometry, J. Mod. Optic. 49, 2325 (2002).

[13] D. Wineland, J. Bollinger, W. Itano, F. Moore, and D. Heinzen, Spin squeezing and reduced quantum noise in spectroscopy, Phys. Rev. A 46, R6797 (1992).

[14] K. McKenzie, D. A. Shaddock, D. E. McClelland, B. C. Buchler, and P. K. Lam, Experimental demonstration of a squeezing-enhanced power-recycled michelson interferometer for gravitational wave detection, Phys. Rev. Lett. 88, 231102 (2002).

[15] J. Bollinger, W. M. Itano, D. Wineland, and D. Heinzen, Optimal frequency measurements with maximally correlated states, Phys. Rev. A 54, R4649 (1996).

[16] D. Leibfried, M. Barrett, T. Schaetz, J. Britton, J. Chiaverini, W. Itano, J. Jost, C. Langer, and D. Wineland, Toward heisenberg-limited spectroscopy with multiparticle entangled states, Science 304, 1476 (2004).

[17] V. Giovannetti, S. Lloyd, and L. Maccone, Quantum-enhanced measurements: beating the standard quantum limit, Science 306, 1330 (2004).

[18] S. F. Huelga, C. Macchiavello, T. Pellizzari, A. K. Ekert, M. B. Plenio, and J. I. Cirac, Improvement of frequency standards with quantum entanglement, Phys. Rev. Lett. 79, 3865 (1997).

[19] D. W. Berry and H. M. Wiseman, Optimal states and almost optimal adaptive measurements for quantum interferometry, Phys. Rev. Lett. 85, 5098 (2000).

[20] M. de Burgh and S. D. Bartlett, Quantum methods for clock synchronization: Beating the standard quantum limit without entanglement, Phys. Rev. A 72, 042301 (2005).

[21] A. Fujiwara and H. Imai, A fibre bundle over manifolds of quantum channels and its application to quantum statistics, J. Phys. A: Math. Theor. 41, 255304 (2008).

[22] R. Demkowicz-Dobrzański, U. Dorner, B. Smith, J. Lundeen, W. Wasilewski, K. Banaszek, and I. Walmsley, Quantum phase estimation with lossy interferometers, Phys. Rev. A 80, 013825 (2009).

[23] B. Escher, R. de Matos Filho, and L. Davidovich, General framework for estimating the ultimate precision limit in noisy quantum-enhanced metrology, Nat. Phys. 7, 406 (2011).

[24] R. Demkowicz-Dobrzański, J. Kołodyński, and M. Guţă, The elusive heisenberg limit in quantum-enhanced metrology, Nat. Commun. 3, 1063 (2012).

[25] J. Kołodyński and R. Demkowicz-Dobrzański, Efficient tools for quantum metrology with uncorrelated noise, New J. Phys. 15, 073043 (2013). 
[26] S. I. Knysh, E. H. Chen, and G. A. Durkin, True limits to precision via unique quantum probe, arXiv:1402.0495 (2014).

[27] R. Demkowicz-Dobrzański and L. Maccone, Using entanglement against noise in quantum metrology, Phys. Rev. Lett. 113, 250801 (2014).

[28] E. M. Kessler, I. Lovchinsky, A. O. Sushkov, and M. D. Lukin, Quantum error correction for metrology, Phys. Rev. Lett. 112, 150802 (2014).

[29] W. Dür, M. Skotiniotis, F. Froewis, and B. Kraus, Improved quantum metrology using quantum error correction, Phys. Rev. Lett. 112, 080801 (2014).

[30] R. Ozeri, Heisenberg limited metrology using quantum error-correction codes. arXiv:1310.3432 (2013).

[31] G. Arrad, Y. Vinkler, D. Aharonov, and A. Retzker, Increasing sensing resolution with error correction, Phys. Rev. Lett. 112, 150801 (2014).

[32] T. Unden, P. Balasubramanian, D. Louzon, Y. Vinkler, M. B. Plenio, M. Markham, D. Twitchen, A. Stacey, I. Lovchinsky, A. O. Sushkov, et al., Quantum metrology enhanced by repetitive quantum error correction, Phys. Rev. Lett. 116, 230502 (2016).

[33] F. Reiter, A. S. Sørensen, P. Zoller, and C. A. Muschik, Dissipative quantum error correction and application to quantum sensing with trapped ions, Nat. Commun. 8, 1822 (2017).

[34] P. Sekatski, M. Skotiniotis, J. Kołodyński, and W. Dür, Quantum metrology with full and fast quantum control, Quantum 1, 27 (2017).

[35] R. Demkowicz-Dobrzański, J. Czajkowski, and P. Sekatski, Adaptive quantum metrology under general markovian noise, Phys. Rev. X 7, 041009 (2017).

[36] S. Zhou, M. Zhang, J. Preskill, and L. Jiang, Achieving the heisenberg limit in quantum metrology using quantum error correction, Nat. Commun. 9, 78 (2018).

[37] D. Layden and P. Cappellaro, Spatial noise filtering through error correction for quantum sensing, npj Quantum Inf. 4, 30 (2018).

[38] D. Layden, S. Zhou, P. Cappellaro, and L. Jiang, Ancilla-free quantum error correction codes for quantum metrology, Phys. Rev. Lett. 122, 040502 (2019).

[39] T. Kapourniotis and A. Datta, Fault-tolerant quantum metrology, Phys. Rev. A 100, 022335 (2019).

[40] K. C. Tan, S. Omkar, and H. Jeong, Quantum-error-correction-assisted quantum metrology without entanglement, Phys. Rev. A 100, 022312 (2019).

[41] S. Zhou and L. Jiang, The theory of entanglement-assisted metrology for quantum channels, arXiv:2003.10559 (2020).

[42] Y. Chen, H. Chen, J. Liu, Z. Miao, and H. Yuan, Fluctuation-enhanced quantum metrology, arXiv:2003.13010 (2020).

[43] T. Baumgratz and A. Datta, Quantum enhanced estimation of a multidimensional field, Phys. Rev. Lett. 116, 030801 (2016).

[44] M. Tsang, R. Nair, and X.-M. Lu, Quantum theory of superresolution for two incoherent optical point sources, Phys. Rev. X 6, 031033 (2016).

[45] P. C. Humphreys, M. Barbieri, A. Datta, and I. A. Walmsley, Quantum enhanced multiple phase estimation, Phys. Rev. Lett. 111, 070403 (2013).

[46] M. Gessner, L. Pezzè, and A. Smerzi, Sensitivity bounds for multiparameter quantum metrology, Phys. Rev. Lett. 121, 130503 (2018).

[47] M. Tsang, H. M. Wiseman, and C. M. Caves, Fundamental quantum limit to waveform estimation, Phys. Rev. Lett. 106, 090401 (2011).

[48] D. W. Berry, M. J. W. Hall, and H. M. Wiseman, Stochastic heisenberg limit: Optimal estimation of a fluctuating phase, Phys. Rev. Lett. 111, 113601 (2013).

[49] K. Matsumoto, A new approach to the cramér-rao-type bound of the pure-state model, J. Phys. A.: Math. Theor. 35, 3111 (2002).

[50] M. G. Genoni, M. G. A. Paris, G. Adesso, H. Nha, P. L. Knight, and M. S. Kim, Optimal estimation of joint parameters in phase space, Phys. Rev. A 87, 012107 (2013).

[51] S. Ragy, M. Jarzyna, and R. Demkowicz-Dobrzański, Compatibility in multiparameter quantum metrology, Phys. Rev. A 94, 052108 (2016).

[52] H. Yuan, Sequential feedback scheme outperforms the parallel scheme for hamiltonian parameter estimation, Phys. Rev. Lett. 117, 160801 (2016). 
[53] N. Kura and M. Ueda, Finite-error metrological bounds on multiparameter hamiltonian estimation, Phys. Rev. A 97, 012101 (2018).

[54] J. Liu and H. Yuan, Control-enhanced multiparameter quantum estimation, Phys. Rev. A 96, 042114 (2017).

[55] R. Nichols, P. Liuzzo-Scorpo, P. A. Knott, and G. Adesso, Multiparameter gaussian quantum metrology, Phys. Rev. A 98, 012114 (2018).

[56] W. Ge, K. Jacobs, Z. Eldredge, A. V. Gorshkov, and M. Foss-Feig, Distributed quantum metrology with linear networks and separable inputs, Phys. Rev. Lett. 121, 043604 (2018).

[57] S. L. Braunstein and C. M. Caves, Statistical distance and the geometry of quantum states, Phys. Rev. Lett. 72, 3439 (1994).

[58] C. W. Helstrom, Quantum detection and estimation theory (Academic press, 1976).

[59] A. S. Holevo, Probabilistic and Statistical Aspects of Quantum Theory (North Holland, Amsterdam, 1982).

[60] R. Demkowicz-Dobrzanski, W. Gorecki, and M. Guta, Multi-parameter estimation beyond quantum fisher information, Journal of Physics A: Mathematical and Theoretical (2020).

[61] H. Nagaoka and M. Hayashi, Asymptotic Theory of Quantum Statistical Inference (World Scientific Singapore, 2005) Chap. 8.

[62] J. Suzuki, Explicit formula for the holevo bound for two-parameter qubit-state estimation problem, J. Math. Phys. 57, 042201 (2016).

[63] M. Guţă and A. Jenčová, Local asymptotic normality in quantum statistics, Comm. Math. Phys. 276, 341 (2007).

[64] K. Yamagata, A. Fujiwara, R. D. Gill, et al., Quantum local asymptotic normality based on a new quantum likelihood ratio, Ann. Statist. 41, 2197 (2013).

[65] A. Fujiwara, Multi-parameter pure state estimation based on the right logarithmic derivative, Math. Eng. Tech. Rep 94, 94 (1994).

[66] F. Albarelli, J. F. Friel, and A. Datta, Evaluating the holevo cramér-rao bound for multiparameter quantum metrology, Phys. Rev. Lett. 123, 200503 (2019).

[67] G. Lindblad, On the generators of quantum dynamical semigroups, Comm. Math. Phys. 48, 119 (1976).

[68] V. Gorini, A. Kossakowski, and E. C. G. Sudarshan, Completely positive dynamical semigroups of n-level systems, J. Math. Phys. 17, 821 (1976).

[69] H.-P. Breuer, F. Petruccione, et al., The theory of open quantum systems (Oxford University Press on Demand, 2002).

[70] S. M. Kay, Fundamentals of statistical signal processing: estimation theory (Prentice Hall, 1993).

[71] R. Gill and S. Massar, State estimation for large ensembles, Phys. Rev. A 61, 042312 (2000).

[72] E. Knill and R. Laflamme, Theory of quantum error-correcting codes, Phys. Rev. A 55, 900 (1997).

[73] M. Grant and S. Boyd, Cvx: Matlab software for disciplined convex programming, .

[74] S. Zhou and L. Jiang, Optimal approximate quantum error correction for quantum metrology, Phys. Rev. Research 2, 013235 (2020).

[75] W. Górecki, R. Demkowicz-Dobrzański, H. M. Wiseman, and D. W. Berry, $\pi$-corrected heisenberg limit, Phys. Rev. Lett. 124, 030501 (2020).

[76] D. A. Lidar, I. L. Chuang, and K. B. Whaley, Decoherence-free subspaces for quantum computation, Phys. Rev. Lett. 81, 2594 (1998). 